\title{
Induction rules, reflection principles, and provably recursive functions
}

\author{
Lev D. Beklemishev* \\ Steklov Mathematical Institute \\ Vavilova 42, 117966 Moscow, RUSSIA \\ e-mail: lev@bekl.mian.su \\ October 10, 1995
}

\begin{abstract}
A well-known result of D. Leivant states that, over basic Kalmar elementary arithmetic $E A$, the induction schema for $\Sigma_{n}$ formulas is equivalent to the uniform reflection principle for $\Sigma_{n+1}$ formulas. We show that fragments of arithmetic axiomatized by various forms of induction rules admit a precise axiomatization in terms of reflection principles as well. Thus, the closure of $E A$ under the induction rule for $\Sigma_{n}$ (or $\left.\Pi_{n+1}\right)$ formulas is equivalent to $\omega$ times iterated $\Sigma_{n}$ reflection principle. Moreover, for $k<\omega$, $k$ times iterated $\Sigma_{n}$ reflection principle over $E A$ precisely corresponds to the extension of $E A$ by $\leq k$ nested applications of $\Sigma_{n}$ induction rule.

The above relationship holds in greater generality than just stated. In fact, we give general formulas characterizing in terms of iterated reflection principles the extension of any given theory (containing $E A$ ) by $\leq k$ nested applications of $\Sigma_{n}$ or $\Pi_{n}$ induction rules. In particular, the closure of a theory $T$ under just one application of $\Sigma_{1}$ induction rule is equivalent to $T$ together with $\Sigma_{1}$ reflection principle for each finite $\Pi_{2}$ axiomatized subtheory of $T$.

These results have closely parallel ones in the theory of subrecursive hierarchies. The analogy can be roughly formulated as follows: counting applications of $\Sigma_{1}$ induction rule in a proof $=$ counting operators of primitive recursion in a primitive recursive program. For example, the above characterization of $\Sigma_{1}$ induction rule implies that, over a class of functions containing elementary ones, one application of nested recursion on $\omega$ can be reduced to one application of primitive recursion and composition. This statement is a slightly refined version of an old result of R. Peter.
\end{abstract}

*The research described in this publication was made possible in part by Grant No. NFQ000 from the International Science Foundation and by the Russian Foundation for Fundamental Research (project 93-011-16015). 


\section{Introduction}

It is well known that first order theories can be defined, over first order logic, by sets of axioms as well as by sets of rules. An axiom can be viewed as a particular kind of rule with an empty, or with some fixed, trivial premise. Vice versa, for a theory $T$ axiomatized by rules, all theorems of $T$ constitute a trivial axiomatization of $T$ by a set of axioms. So, if one identifies a theory with its set of provable theorems - a point of view supported by the model-theoretic tradition in logic - there is no essential difference between rules and axioms.

Recently, a new interest was aroused in proof-theory towards the questions of a more "intensional", syntax-depending character. One can mention the active research being done in the area of complexity of proofs; propositional proof systems (cf, e.g., [5]); investigations on so-called Kreisel's Conjecture (cf, e.g.,[1]). These developments are mainly inspired by potential applications in Theoretical Computer Science, rather than by purely foundational questions. On the other hand, they are very essential for proof theory itself, for in all these areas the existing proof-theoretic techniques cannot be completely replaced by the alternative model-theoretic ones, because of the intensional character of problems.

This paper is devoted to a detailed proof-theoretic analysis of restricted induction rules in arithmetic. Our main results characterize closures of arithmetical theories containing $E A$ by induction rules in terms of axioms. These results have intensional character in the sense that we are looking for natural and informative axiomatizations, rather than for easy but useless ones.

Although logicians usually have a very good intuition about particular axiomatizations, the general question "What is a natural axiomatization of a formal theory?" is somewhat vague from strictly mathematical point of view. ${ }^{1}$ In our case we have both informal and formal evidence to the effect that axiomatizations by rules and their equivalent characterizations by axioms (reflection principles) are 'naturally related' to each other:

(a) Reflection principles are well-studied schemata in proof theory, with a clear (meta)mathematical meaning.

(b) Our characterizations are informative in the sense that they yield several interesting corollaries concerning finite (non)axiomatizability of theories given by induction rules, optimal complexity of their axiomatizations, and give wide sufficient conditions for the equivalence of (closures of theories by) $\Pi_{n+1}$ and $\Sigma_{n}$ induction rules. Besides, they allow to give new proofs of several old 'extensional' results, such as the conservativity results for induction schemata over induction rules, characterizations of provably (total) recursive functions of theories axiomatized by rules, Peter's theorem on nested recursion, and others.

(c) The structure of the axiomatizations by iterated reflection principles pre-

\footnotetext{
${ }^{1}$ There are other well-known open questions in proof theory of similar nature, e.g., "What is a natural system of ordinal notation?".
} 
cisely corresponds to the structure of nestings of applications of induction rules.

The latter, more formal, kind of evidence will be carefully explained below. For further discussion we must fix some terminology and formulate a few background results.

Kalmar elementary arithmetic $E A$ is a theory known in several equivalent formulations. When formulated in the standard language of Peano arithmetic $P A$ it has the name $I \Delta_{0}+$ EXP and is axiomatized by restricting, in the standard formulation of $P A$, the schema of induction

$$
A(0) \wedge \forall x(A(x) \rightarrow A(x+1)) \rightarrow \forall x A(x)
$$

to bounded formulas $A(x)$ and by adding a $\Pi_{2}$ axiom stating that the function $2^{x}$ is total. It is well-known that $I \Delta_{0}+$ EXP is a finitely axiomatizable theory $[3]$.

In an alternative formulation, the language of $E A$ contains function symbols for all Kalmar elementary functions, and mathematical axioms of $E A$ are (1) (open) defining equations for all these functions; (2) the schema of induction for open formulas. It is known that $E A$ admits a purely universal (or quantifier free) axiomatization in this language. The two formulations of $E A$ are equivalent in the sense that the second theory can be viewed as a conservative 'extension by definitions' of the first one.

Switching between the two different axiomatizations of $E A$ is technically useful and will be exploited below. Let us also mention the fact that there exists a finite, purely universal formulation of $E A$ in a language with symbols for finitely many elementary functions. This fact is closely related to a well-known theorem, originally due to Rödding, stating that the class of Kalmar elementary functions has a finite basis under composition (see, e.g., $[8,10]$ ). We shall sketch a proof of this useful fact, as well as that of finite basis theorem, in Section 4.

It is known that $E A$ is strong enough to reasonably formalize syntax, provability, Gödel's incompleteness theorems, partial truthdefinitions (see [3]). In this paper we take $E A$ as a base theory, that is, all theories considered below are assumed to contain $E A$.

C. Parsons was probably the first to systematically study fragments of $P A$ obtained by restricting various forms of induction to classes of the arithmetic hierarchy. In $[11,12]$, among other things, he showed that, over $E A$, the induction schema (1) for $\Sigma_{n}$ formulas $A(x)$, denoted $\Sigma_{n}$-IA, is strictly stronger than the corresponding induction rule for $\Sigma_{n}$ formulas, $\Sigma_{n}$-IR:

$$
A(0), \forall x(A(x) \rightarrow A(x+1)) \vdash \forall x A(x) .
$$

Parsons demonstrated that many other natural forms of restricted induction over $E A$ are equivalent to one of these two. In particular, for $n \geq 1$,

$$
E A+\Sigma_{n}-\mathrm{IA} \equiv E A+\Pi_{n}-\mathrm{IA},
$$


(this theory is also often denoted $I \Sigma_{n}$ ) and

$$
E A+\Sigma_{n} \text {-IR } \equiv E A+\Pi_{n+1} \text {-IR. }
$$

Here the expression $T \equiv U$ means that the theories $T$ and $U$ are deductively equivalent, i.e., have the same set of theorems.

Despite the two results looking very similar, they are rather different in nature, as the reader familiar with their proofs undoubtedly feels. There are more formal reasons for such a feeling. Equivalence (2) actually holds over any theory $T$ containing $E A$, and this indicates a really tight relationship between the two axiom schemata. On the other hand, it is well-known that equivalence (3) may cease to be true for some theories stronger than $E A$. For example,

$$
I \Sigma_{1}+\Sigma_{1}-\mathrm{IR} \equiv I \Sigma_{1} \not \equiv I \Sigma_{1}+\Pi_{2}-\mathrm{IR},
$$

because $I \Sigma_{1}+\Pi_{2}$-IR proves the consistency of $I \Sigma_{1}$, e.g., by our results in Section 3. This shows that, from some sharper point of view, $\Sigma_{1}$-IR and $\Pi_{2}$-IR are substantially different rules. In order to accurately formulate this point of view we adopt a few rather general definitions.

Since the rules we deal with in this paper are typically schematic rules, we say that a rule is a set of instances, that is, expressions of the form

$$
\frac{A_{1}, \ldots, A_{n}}{B},
$$

where $A_{1}, \ldots, A_{n}$ and $B$ are formulas. Derivations using rules are defined in the standard way; $T+R$ denotes the closure of a theory $T$ under a rule $R$ and first order logic. $[T, R]$ denotes the closure of $T$ under unnested applications of $R$, that is, the theory axiomatized over $T$ by all formulas $B$ such that, for some formulas $A_{1}, \ldots, A_{n}$ derivable in $T, \frac{A_{1}, \ldots, A_{n}}{B}$ is an instance of $R$.

Definition 1. Let $R_{1}$ and $R_{2}$ be rules. $R_{1}$ is reducible to $R_{2}$ (denoted $R_{1} \leq R_{2}$ ) iff, for every theory $T$ containing $E A,\left[T, R_{1}\right] \subseteq\left[T, R_{2}\right] . R_{1}$ and $R_{2}$ are congruent $\left(R_{1} \cong R_{2}\right)$ iff $R_{1} \leq R_{2}$ and $R_{2} \leq R_{1}$.

Informally, $R_{1} \leq R_{2}$ means that an arbitrary application of $R_{1}$ can be modeled over $E A$ by unnested applications of $R_{2}$. Notice that $\leq$ is reflexive and transitive, so that $\cong$ is an equivalence relation. Also notice that the notion of congruence does not depend on the choice of a base theory. For the purposes of this paper we may safely identify congruent rules.

We say that a rule $R$ is congruent to a set of axioms $U$, iff $R$ is congruent to $U$ considered as a trivial schematic rule (with the empty premise). Equivalently, iff $[T, R] \equiv T+U$ for any theory $T$ extending $E A$. Notice that rules congruent to axiom schemata have a trivial behaviour in the sense that they cannot be applied fruitfully more than once: nested applictions of such rules do not yield new theorems. 
Definition 2. $R_{1}$ is derivable from $R_{2}$ (denoted $R_{1} \preceq R_{2}$ ) iff for any application $\frac{A_{1}, \ldots, A_{n}}{B}$ of $R_{1}$ there exists a derivation of $B$ from $A_{1}, \ldots, A_{n}$ using $E A$ and rule $R_{2}$.

In other words, $R_{1} \preceq R_{2}$ iff for every theory $T$ containing $E A$, $\left[T, R_{1}\right] \subseteq T+R_{2}$. Clearly, $R_{1} \leq R_{2}$ implies $R_{1} \preceq R_{2}$ but not necessarily vice versa. Below we shall see that equivalences of rules established by purely elementary methods can usually be strengthened to congruences. On the other hand, equivalence proofs involving more sophisticated methods usually depend on the choice of a particular base theory and therefore do not yield reducibilities either in the sense of Definition 1 or 2.

Example. We have seen that $\Pi_{2}$-IR $\npreceq \Sigma_{1}$-IR, although the closure of $E A$ under each of these rules is the same. On the other hand, obviously $\Sigma_{1}$-IR $\leq \Pi_{2}$-IR. Corollary 2.1 in Section 2 shows that $\Pi_{n}$-IR $\leq \Sigma_{n}$-IR but not vice versa.

The plan of the paper is as follows. In Section 2 we classify various forms of induction rules modulo congruence relation. We shall show that these rules, most commonly, fall into one of the three distinct categories: (a) rules congruent to induction axiom schemata; (b) rules congruent to $\Sigma_{n}$ induction rule $\Sigma_{n}$-IR; (c) rules congruent to $\Pi_{n}$ induction rule $\Pi_{n}$-IR. (An interesting candidate for falling out of this classification is the induction rule for boolean combinations of $\Sigma_{1}$ formulas, which is derivable from, but possibly not reducible to, $\Sigma_{n}$-IR; see below.)

The question of the axiomatizability of rules of category (a) is trivially settled. So, in the remaining part of the paper we analyze the other two cases. In Section 3 we introduce reflection principles and characterize $\Pi_{n}$-IR. A similar characterization of $\Sigma_{n}$-IR is more difficult and is given in Section 7 for $\Sigma_{1}$-IR, and in Section 8 for $\Sigma_{n}$-IR, $n>1$. The characterization of $\Sigma_{1}$-IR requires a rather careful analysis of provably recursive functions of theories axiomatized by this rule. In Section 4 we recall basic facts about provably recursive functions and formulate an easy characterization of these functions for closures of $\Pi_{2}$ axiomatized theories by $\Sigma_{1}$-IR. In Section 5 we analyze the question, when the class of provably recursive functions of a theory is elementarily closed. A natural sufficient condition is formulated in terms of $\Sigma_{1}$ collection rule. In Section 6, on the basis of these results, we construct a suitable universal function for the class of provably recursive functions of a finite $\Pi_{2}$ axiomatized extension of $E A$ using only unnested applications of $\Sigma_{1}$-IR over that theory. This allows us to obtain in Section 7 the required characterization of $\Sigma_{1}$-IR, and subsequently relativize it to $\Sigma_{n}$-IR for $n>1$.

It should be said that in the proof of our main results we did not try to be overly laconic. We have included proofs of several results which were formally never used in the main proofs, like the above mentioned theorem of R. Peter 
on nested recursion. Or results the use of which could be avoided, like the finite basis theorem for Kalmar elementary functions. It seems to us that proofs of these easy facts (modulo the rest of our techniques) would enhance the reader's general understanding of peculiar phenomena treated in this paper, so we decided to include them. The results of Section 3 of this paper have been earlier announced in $[2]$.

Acknowledgements. Main results of this paper were obtained while I was a guest visitor at the Department of Philosophy, Utrecht University in SeptemberOctober, 1994. Thanks are due to the Logic Group of the Department of Philosophy for providing an excellent research environment.

The paper owes much to participants of the seminar 'Arithmetic Colloquium', particularly to D. de Jongh, A. Visser, V. Shavrukov, R. Verbrugge, D. Zambella. Their questions and remarks helped me to rethink and considerably improve earlier results. I am particularly grateful to Albert Visser who inspired my interest in characterizing fragments of arithmetic by reflection principles. Discussions with Ulrich Kohlenbach of the J.W. Goethe-Universität at Frankfurt have also been very useful.

\section{Basic equivalences}

C. Parsons showed that many natural forms of induction (of restricted arithmetical complexity) over $E A$ are equivalent to either $\Sigma_{n}$-IR or $\Sigma_{n}$-IA. In this section we obtain a few more results of this kind. We classify various forms of induction rules modulo the sharper congruence relation. Some of Parsons' equivalences then turn out to be congruences, whereas some others do not. We also examine a few rules that have not been considered by Parsons. In addition to IR we consider the following forms of induction rule:

$$
\begin{array}{ll}
\mathrm{IR}_{0}: & \forall x(A(x) \rightarrow A(x+1)) \vdash A(0) \rightarrow \forall x A(x) \\
\mathrm{IR}_{<}: & \forall x(\forall y<x A(y) \rightarrow A(x)) \vdash \forall x A(x) \\
\mathrm{LR}: & \exists x A(x) \vdash \exists x(A(x) \wedge \forall y<x \neg A(y))
\end{array}
$$

As usual, for $\Gamma$ a class of arithmetical formulas, $\Gamma$-IR $\mathrm{IR}_{0}, \Gamma-\mathrm{IR}_{<}$, and $\Gamma$-LR will denote the above rules with the restriction that $A \in \Gamma$. We also assume that formulas $A(x)$ may contain free parameters other than $x$.

Proposition 2.1. $\Sigma_{n}-\mathrm{IR}_{0} \cong \Pi_{n}-\mathrm{IR}_{0} \cong \Sigma_{n}$-IR

Proof. 1 . The congruence $\Sigma_{n}-\mathrm{IR}_{0} \cong \Pi_{n}-\mathrm{IR}_{0}$ is proved in analogy with the proof of the equivalence of $\Sigma_{n}$-IA and $\Pi_{n}$-IA (cf [12]). For example, to show that $\Sigma_{n}-\mathrm{IR}_{0} \leq \Pi_{n}-\mathrm{IR}_{0}$ consider a formula $A(x) \in \Sigma_{n}$ such that

$$
T \vdash \forall x(A(x) \rightarrow A(x+1)) .
$$


Then for $B(a, x):=\neg A(a \dot{-} x)$ one has

$$
T \vdash \forall x(B(a, x) \rightarrow B(a, x+1)),
$$

whence

$$
\begin{aligned}
{\left[T, \Pi_{n}-\mathrm{IR}_{0}\right] } & \vdash B(a, 0) \rightarrow \forall x B(a, x) \\
& \vdash B(a, 0) \rightarrow B(a, a) \\
& \vdash A(0) \rightarrow A(a), \quad \text { q.e.d. }
\end{aligned}
$$

Notice that similar trick does not work with the rule IR.

2. Obviously, $\Sigma_{n}$ IR $\leq \Sigma_{n}-\mathrm{IR}_{0}$ and $\Pi_{n}$-IR $\leq \Pi_{n}-\mathrm{IR}_{0}$. So, it only remains to show that $\Sigma_{n}-\mathrm{IR}_{0} \leq \Sigma_{n}$-IR. Let $\exists y A(y, x) \in \Sigma_{n}$ with $A(y, x) \in \Pi_{n-1}$, and let

$$
T \vdash \forall x(\exists y A(y, x) \rightarrow \exists y A(y, x+1)) .
$$

Then we have

$$
T \vdash \forall x(\exists y(A(a, 0) \rightarrow A(y, x)) \rightarrow \exists y(A(a, 0) \rightarrow A(y, x+1)))
$$

and obviously

$$
T \vdash \exists y(A(a, 0) \rightarrow A(y, 0))
$$

It follows that

$$
\begin{aligned}
{\left[T, \Sigma_{n} \text {-IR }\right] } & \vdash \forall x \exists y(A(a, 0) \rightarrow A(y, x)) \\
& \vdash \exists u A(u, 0) \rightarrow \forall x \exists y A(y, x), \quad \text { q.e.d. }
\end{aligned}
$$

Corollary 2.1. $\Pi_{n}$-IR $\leq \Sigma_{n}$-IR.

Proof: via $\Pi_{n}-\mathrm{IR}_{0}$. Also notice that $\Sigma_{n}$-IR $\npreceq \Pi_{n}$-IR, because, for example, $E A+\Sigma_{2}$-IR contains $I \Sigma_{1}$, whereas $E A+\Pi_{2}$-IR, being deductively equivalent to $P R A$, does not.

Proposition 2.2. $\Pi_{n}-\mathrm{IR}_{<} \cong \Pi_{n}-\mathrm{IR}, \Sigma_{n}-\mathrm{IR}_{<} \cong \Sigma_{n}-\mathrm{IR}$

Proof. The only nontrivial reduction is $\Sigma_{n}$-IR $<\leq \Sigma_{n}$-IR. (Notice that, if $A(x) \in \Sigma_{n}$, the formula $\forall y \leq x A(y)$ need not be equivalent to a $\Sigma_{n}$-formula in absense of $\Sigma_{n}$-collection principle, and so the obvious argument does not work.)

Suppose

$$
T \vdash \forall x(\forall y<x A(y) \rightarrow A(x)),
$$

where $A(x):=\exists u A_{0}(x, u), A_{0}(x, u) \in \Pi_{n-1}$. Define:

$$
B(x):=\exists z \forall y \leq x A_{0}\left(y,(z)_{y}\right) .
$$


Here $(z)_{y}$ denotes the $y$-th element of a sequence coded by $z$, the standard coding function being Kalmar elementary. Clearly, $B(x) \in \Sigma_{n}$, and from (4) one readily obtains

$$
T \vdash B(0) \wedge \forall x(B(x) \rightarrow B(x+1)) .
$$

Applying $\Sigma_{n}$-IR once, we get $\forall x B(x)$ and $\forall x \exists y A_{0}(x, u)$, q.e.d.

Now we examine some rules congruent to axiom schemata. The effect of such rules over a theory $T$ is precisely that of adding to $T$ a fixed amount of axioms (that do not depend on $T$ ). This idea is spelled out in the following definition.

Definition 3. A rule $R$ is congruent to a set of formulas $U$ (denoted $R \cong U$ ) iff, for every theory $T$ containing $E A,[T, R] \equiv T+U$.

It is not difficult to see that, if $R \cong U$, then we have

$$
[[T, R], R] \equiv[T, R]+U \equiv(T+U)+U \equiv T+U
$$

and so, such a rule can nontrivially be applied only once. The reader, however, should keep in mind that not every rule with this property is congruent to a set of axioms (for each $T$ the set $U$ may be different). We shall encounter one important rule of this sort, $\Sigma_{1}$-collection rule, later on in the paper. Also notice that in order to demontstrate $R \cong U$ it is enough to check that $[E A, R]$ contains $U$ and that $T+U$ is closed under $R$ for every theory $T$.

Of the rules congruent to axiom schemes the most obvious one is the usual Gentzen-style rule of induction, which can also be called "the induction rule with side formulas". In Hilbert-style formulation it may look, e.g., as follows:

$$
\frac{B \rightarrow \forall x(A(x) \rightarrow A(x+1))}{B \rightarrow(A(0) \rightarrow \forall x A(x))}
$$

It is well-known that, whenever the complexity of the formula $A$ is restricted to, say, $\Sigma_{n}$, this rule provides an alternative axiomatization of $I \Sigma_{n}$ (over $E A$ ). Moreover, the reader may easily check that to derive an instance of $\Sigma_{n}$-IA only one application of the rule is necessary. On the other hand, the fact that $T+\Sigma_{n}$-IA is closed under the induction rule with side formulas is obvious, hence the rule is congruent to $\Sigma_{n}$-IA. Of course, such an effect is only possible because no restriction was imposed on the arithmetical complexity of the 'side formula' $B$. Our further examples are of a somewhat more delicate nature.

Recall that, for a class of arithmetical formulas $\Gamma, \Delta_{0}(\Gamma)$-formulas are those obtained from $\Gamma$ by means of boolean connctives and bounded quantifiers. Parsons [12] essentially proved the following fact.

Proposition 2.3. $\Delta_{0}\left(\Sigma_{n}\right)-\mathrm{IR} \cong \Sigma_{n}$-IA 
Proof. To derive an instance of $\Sigma_{n}$-IA apply IR to the following $\Delta_{0}\left(\Sigma_{n}\right)$ formula:

$$
A(0) \wedge \forall x<a(A(x) \rightarrow A(x+1)) \rightarrow \forall x \leq a A(x),
$$

where $A(x) \in \Sigma_{n}$.

To show that $T+\Sigma_{n}$-IA is closed under $\Delta_{0}\left(\Sigma_{n}\right)$-IR for each theory $T$ notice that an even stronger fact is known: $I \Sigma_{n}$ contains $\Delta_{0}\left(\Sigma_{n}\right)$-IA (cf [12] or [3], Lemma 2.14, p. 65), q.e.d.

The above proposition has a somewhat paradoxical consequence that $\Delta_{0}\left(\Sigma_{1}\right)$ IR turns out to be actually stronger than $\Pi_{2}$-IR over $E A$. This looks strange because we all are used to the fact that in the standard model of arithmetic $\Delta_{0}\left(\Sigma_{1}\right)$ sets are $\Delta_{2}$ and hence strictly lower in the hierarchy than $\Pi_{2}$ sets. No contradiction in mathematics arises from this because $E A$ is a weak enough theory to think (or rather, not to exclude) that $\Delta_{0}\left(\Sigma_{1}\right)$ sets can be very complex. In fact, Proposition 2.3 provides a relevant instance of $\Sigma_{1}$-IA of the form (5) as an example to this effect. Now we are ready to examine the least element rule LR.

Proposition 2.4. $\Pi_{n}-\mathrm{LR} \cong \Delta_{0}\left(\Sigma_{n}\right)-\mathrm{LR} \cong \Sigma_{n}-\mathrm{LR}$

Proof. 1. The first congruence is proved very similarly to the quoted Lemma 2.14 of [3]. We only sketch the argument.

For a formula $A(\vec{x}):=A\left(x_{1}, \ldots, x_{k}\right)$ let " $q$ is a $z$-piece of $A$ " denote the following formula:

" $q$ codes a function $[0 ; z]^{k} \rightarrow\{0,1\} " \wedge \forall x_{1}, \ldots, x_{k} \leq z(A(\vec{x}) \leftrightarrow " q(\vec{x})=1 ")$.

We say that $A$ is piecewise coded in a theory $T$ iff

$$
T \vdash \forall z \exists q \text { " } q \text { is a } z \text {-piece of } A \text { ". }
$$

It is readily seen that the class of formulas piecewise coded in a theory $T$ containing $E A$ is closed under boolean connectives and bounded quantifiers.

Now we show that the theory $\left[E A, \Pi_{n}-\mathrm{LR}\right]$ piecewise encodes all $\Sigma_{n}$-formulas. Indeed, for any such formula $A(\vec{x})$ we obviously have

$$
E A \vdash \exists q\left(q:[0 ; a]^{k} \rightarrow\{0,1\} \wedge \forall \vec{x} \leq a(A(\vec{x}) \rightarrow q(\vec{x})=1)\right),
$$

because, e.g., one may take for $q$ the function identically equal to 1 . Applying $\Pi_{n}$-LR once we get the minimal such $q$. It faithfully encodes the $a$-piece of $A$ because the standard coding of finite functions has the property that functions with smaller values are assigned smaller codes. It follows that all $\Sigma_{n}$, and hence all $\Delta_{0}\left(\Sigma_{n}\right)$, formulas are piecewise coded in $\left[E A, \Pi_{n}\right.$-LR].

Now it is easy to derive $\Delta_{0}\left(\Sigma_{n}\right)$-LR. Let $E A \vdash \exists x A(x)$, where $A(x) \in \Delta_{0}\left(\Sigma_{n}\right)$. Then we have:

$$
\left[E A, \Pi_{n}-\mathrm{LR}\right] \vdash \exists x, q(A(x) \wedge \text { " } q \text { is a } x \text {-piece of } A \text { " }) .
$$


For this $q$, using only elementary induction we can find the minimal $x$ such that $q(x)=1$. It coincides with the least $x$ such that $A(x)$ holds since $q$ is the $x$-piece of $A$.

2. To demonstrate the second congruence it is sufficient to show that every $\Pi_{n}$ formula is piecewise coded in $\left[E A, \Sigma_{n}\right.$-LR]. Let $\forall u A_{0}(u, x)$ be such a formula, with $A_{0} \in \Sigma_{n-1}$. We obviously have

$$
E A \vdash \exists q \exists u \forall x \leq a\left(A_{0}\left((u)_{x}, x\right) \rightarrow q(x)=1\right) .
$$

Using $\Sigma_{n}$-LR pick the least such $q$. It is not difficult to see that $q$ is as required, q.e.d.

Proposition 2.5. $\Pi_{n^{-}} \mathrm{LR} \cong \Sigma_{n^{-}} \mathrm{LR} \cong \Sigma_{n^{-}} \mathrm{IA}$

Proof. It is well-known that $\Sigma_{n}$-IA is equivalent to the least number principle for $\Delta_{0}\left(\Sigma_{n}\right)$ formulas (cf [3]), hence $T+\Sigma_{n}$-IA is closed under $\Delta_{0}\left(\Sigma_{n}\right)$-LR for any theory $T$. Now we derive the least number principle for an arbitrary $\Delta_{0}\left(\Sigma_{n}\right)$ formula $A(x)$. Obviously,

$$
E A \vdash \exists x(A(a) \rightarrow A(x))
$$

Using Proposition 2.4 we conclude that $\left[E A, \Pi_{n}\right.$-LR] contains

$$
\exists x((A(a) \rightarrow A(x)) \wedge \forall y<x \neg(A(a) \rightarrow A(y))) .
$$

This formula implies

$$
\exists x(A(a) \rightarrow(A(x) \wedge \forall y<x \neg A(y)))
$$

and

$$
\exists z A(z) \rightarrow \exists x(A(x) \wedge \forall y<x \neg A(y))),
$$

q.e.d.

The last rule we shall consider in this section is the induction rule for boolean combinations of $\Sigma_{n}$ formulas, $\mathcal{B}\left(\Sigma_{n}\right)$-IR. This rule is of interest because it is derivable from $\Sigma_{n}$-IR, as we shall see below, but, most probably, is not reducible to it. The following lemma is well-known and can be proved by purely propositional manipulations.

Lemma 2.1. Every $\mathcal{B}\left(\Sigma_{n}\right)$ formula is logically equivalent to a formula of the form

$$
\pi_{1} \wedge \neg\left(\pi_{2} \wedge \neg\left(\pi_{3} \wedge \ldots \wedge \neg \pi_{k}\right) \ldots\right),
$$

for suitable $\Pi_{n}$ formulas $\pi_{1}, \ldots, \pi_{k}$. 
We say that formulas of the form $(*)$ and their negations have rank $k$. More precisely, rank of a $\mathcal{B}\left(\Sigma_{n}\right)$ formula is the length of its shortest representation in the form $(*)$ or in the negated form. Thus, $\Sigma_{n}$ and $\Pi_{n}$ formulas, and only these formulas, have rank 1 . Let $\mathcal{B}_{k}\left(\Sigma_{n}\right)$ denote the class of all formulas of rank $\leq k$.

Proposition 2.6. $\mathcal{B}\left(\Sigma_{n}\right)-\mathrm{IR}_{0} \preceq \Sigma_{n}-\mathrm{IR}_{0}$

Proof. We shall show by induction on $k$ that

$$
\mathcal{B}_{k}\left(\Sigma_{n}\right)-\mathrm{IR}_{0} \preceq \Sigma_{n}-\mathrm{IR}_{0}
$$

for all $k$. For $k=1$ the statement follows from Proposition 2.1. Since an application of $\mathrm{IR}_{0}$ for a formula $\neg A(x)$ is reducible to $\mathrm{IR}_{0}$ for $A(a-x)$, as in the proof of Proposition 2.1, for the induction step it will be sufficient to treat formulas of the form $(*)$ only.

Assuming that proposition holds for all boolean combinations of rank $<k$, consider a formula $A(x)$ of the form $(*)$ such that a theory $T$ proves

$$
\forall x(A(x) \rightarrow A(x+1))
$$

Define:

$$
\begin{aligned}
\pi(x) & :=\forall y \leq x \pi_{1}(y) \\
A_{1}(x) & :=\neg\left(\pi_{2} \wedge \neg\left(\pi_{3} \wedge \ldots \neg \pi_{k}\right) \ldots\right) .
\end{aligned}
$$

First we shall show that $T$ proves

$$
\forall x\left(\left(\pi(x) \rightarrow A_{1}(x)\right) \rightarrow\left(\pi(x+1) \rightarrow A_{1}(x+1)\right)\right)
$$

To this end we derive $A_{1}(x+1)$ from two assumptions: $\pi(x) \rightarrow A_{1}(x)$ and $\pi(x+1)$.

$\begin{array}{lll}\text { 1. } & \pi(x+1) & \text { (assumption) } \\ \text { 2. } & \pi(x) \rightarrow A_{1}(x) & \text { (assumption) } \\ \text { 3. } & \pi_{1}(x) & \text { (from } 1) \\ \text { 4. } & \pi(x) & (\text { from } 1) \\ \text { 5. } & A_{1}(x) & (\text { from } 2,4) \\ \text { 6. } & \pi_{1}(x) \wedge A_{1}(x) & \text { (from } 3,5) \\ \text { 7. } & \pi_{1}(x+1) \wedge A_{1}(x+1) & \text { (by } 6 \text { and }(6)) \\ \text { 8. } & A_{1}(x+1) & \text { (by } 7),\end{array}$

and this proves (7).

Now we notice that the formula $\pi(x) \rightarrow A_{1}(x)$ is logically equivalent to

$$
\neg\left(\left(\pi \wedge \pi_{2}\right) \wedge \neg\left(\pi_{3} \wedge \ldots \wedge \neg \pi_{k}\right) \ldots\right),
$$


that is, has rank $\leq k-1$. By the induction hypothesis we may conclude that the formula

$$
\left(\pi(0) \rightarrow A_{1}(0)\right) \rightarrow \forall x\left(\pi(x) \rightarrow A_{1}(x)\right) .
$$

is provable in $T+\Sigma_{n}-\mathrm{IR}_{0}$. It follows that so is the formula

$$
A_{1}(0) \rightarrow \forall x\left(\pi(x) \rightarrow A_{1}(x)\right) .
$$

Since the formula $A_{1}$ has the form $\neg\left(\pi_{2} \wedge A_{2}\right)$, where $A_{2}$ has rank $\leq k-2$, from (8) we conclude

$$
\begin{aligned}
& \neg \pi_{2}(0) \rightarrow \forall x\left(\pi(x) \rightarrow A_{1}(x)\right), \\
& \neg A_{2}(0) \rightarrow \forall x\left(\pi(x) \rightarrow A_{1}(x)\right) .
\end{aligned}
$$

Now we shall derive

$$
\forall x\left(\left(\neg \pi_{2}(0) \rightarrow \pi(x)\right) \rightarrow\left(\neg \pi_{2}(0) \rightarrow \pi(x+1)\right)\right)
$$

in $T+\Sigma_{n}-\mathrm{IR}_{0}$. Derivation:

$$
\begin{array}{lll}
\text { 1. } & \neg \pi_{2}(0) \rightarrow \pi(x) & \text { (assumption) } \\
\text { 2. } \neg \pi_{2}(0) & \text { (assumption) } \\
\text { 3. } \pi(x) & (1,2) \\
\text { 4. } & \pi(x) \rightarrow A_{1}(x) & ((9), 2) \\
\text { 5. } & A_{1}(x) & \\
\text { 6. } & \pi_{1}(x) \wedge A_{1}(x) & (3,5) \\
\text { 7. } & \pi_{1}(x+1) \wedge A_{1}(x+1) & (\text { by }(6)) \\
\text { 8. } & \pi(x+1) & (7,3)
\end{array}
$$

Since $\neg \pi_{2}(0) \rightarrow \pi(x)$ is a $\Pi_{n}$ formula, by $\mathrm{IR}_{0}$ using Proposition 2.1 we obtain

$$
\left(\neg \pi_{2}(0) \rightarrow \pi(0)\right) \rightarrow\left(\neg \pi_{2}(0) \rightarrow \forall x \pi(x)\right),
$$

and hence

$$
\left(\neg \pi_{2}(0) \wedge \pi(0)\right) \rightarrow \forall x \pi(x) .
$$

Similarly, using (10) and induction on

$$
\neg A_{2}(0) \rightarrow \pi(x),
$$

we can derive

$$
\left(\neg A_{2}(0) \wedge \pi(0)\right) \rightarrow \forall x \pi(x) .
$$

(It has to be noticed that the rank of formula (12) is at worst $(k-2)+1=k-1$, so the induction hypothesis (6) is applicable.)

Putting (11) and (13) together we may conclude that $T+\Sigma_{n}-\mathrm{IR}_{0}$ proves

$$
\left(\pi(0) \wedge\left(\neg \pi_{2}(0) \vee \neg A_{2}(0)\right)\right) \rightarrow \forall x \pi(x),
$$


that is,

$$
\left(\pi(0) \wedge A_{1}(0)\right) \rightarrow \forall x \pi(x),
$$

and together with (8) this yields

$$
\left(\pi_{1}(0) \wedge A_{1}(0)\right) \rightarrow\left(\forall x \pi_{1}(x) \wedge \forall x A_{1}(x)\right.
$$

as required, q.e.d.

Corollary 2.2. $\mathcal{B}\left(\Sigma_{n}\right)$-IR $\preceq \Sigma_{n}$-IR

Proof: Obviously $\mathcal{B}\left(\Sigma_{n}\right)$-IR and $\mathcal{B}\left(\Sigma_{n}\right)-\mathrm{IR}_{0}$ are congruent.

Remark 2.1. From the given proof of Proposition 2.6 it is apparent that we used many nested applications of $\Sigma_{n}-\mathrm{IR}_{0}$ in order to model a single application of $\mathcal{B}\left(\Sigma_{n}\right)-\mathrm{IR}_{0}$. The number of nestings is a function of the rank $k$ of boolean combinations of $\Sigma_{n}$-formulas. In fact, this function (let us temporarily call it $f(k))$ can be precisely estimated:

$$
\left\{\begin{array}{l}
f(2 k)=2 \cdot 3^{k-1} \\
f(2 k+1)=4 \cdot 3^{k-1}
\end{array}\right.
$$

Initial values of $f$ are $1,2,4,6,12,18,36, \ldots$; notice that $f$ grows exponentially. I do not know if this result is optimal, that is, if the exact values of $f$ can be decreased. Later, when analysing the relationship between $\Pi_{n+1}$ and $\Sigma_{n}$ induction rules, we shall find out that for a certain class of base theories $T$ the exponential number of applications of $\Sigma_{n}$-IR can dramatically be reduced to a single application, but I do not know if this is true for arbitrary theories.

Open question: is $\mathcal{B}\left(\Sigma_{n}\right)$-IR congruent to $\Sigma_{n}$ IR?

\section{$3 \quad \Pi_{n}$ induction rule}

In this section we give a characterization of $\Pi_{n}$-IR in terms of iterated reflection principles.

Reflection principles, for an r.e. arithmetical theory $T$, are formal schemata expressing the soundness of $T$, that is, the statement that "every sentence provable in $T$ is true". More precisely, if $\operatorname{Prov}_{T}(x)$ denotes the canonical $\Sigma_{1}$ provability predicate for $T$, then the (uniform) reflection principle for $T$ is the schema

$$
\forall x\left(\operatorname{Prov}_{T}(\ulcorner A(\dot{x})\urcorner) \rightarrow A(x)\right),
$$

for all formulas $A(x)$. This schema is denoted $\operatorname{RFN}(T)$. Partial reflection principles are obtained from it by imposing a restriction that the formula $A$ may only range over a certain subclass $\Gamma$ of the class of $T$-formulas. Such schemata will 
be denoted $\operatorname{RFN}_{\Gamma}(T)$, and for $\Gamma$ one usually takes one of the classes $\Sigma_{n}$ or $\Pi_{n}$ of the arithmetical hierarchy. The following two basic facts on uniform reflection principles are well-known (cf [21]) and easy:

1) $\operatorname{RFN}_{\Sigma_{n}}(T)$ is equivalent to $\operatorname{RFN}_{\Pi_{n+1}}(T)$ over $E A$, for $n \geq 1$. $\operatorname{RFN}_{\Pi_{1}}(T)$ is equivalent to $\operatorname{Con}(T)$, the consistency assertion for $T$.

2) The schema $\operatorname{RFN}_{\Pi_{n}}(T)$ is equivalent to a single $\Pi_{n}$ sentence (over $E A$ ). This essentially follows from the existence of partial truthdefinitions.

A old and well-known result of Kreisel and Lévy [6] says that an alternative axiomatization of Peano Arithmetic over $E A$ can be obtained by replacing the induction schema by the full uniform reflection principle for $E A$ :

$$
P A \equiv E A+\operatorname{RFN}(E A) \text {. }
$$

D. Leivant sharpened this result by showing that the hierarchies of restricted induction schemata and restricted reflection principles over $E A$ actually coincide:

$$
I \Sigma_{n} \equiv E A+\operatorname{RFN}_{\Sigma_{n+1}}(E A) .
$$

Here we establish a precise relationship between the $\Pi_{n}$ induction rule and the hierarchy of iterated reflection principles.

All theories in this section are formulated in the standard language of $P A$. $I \Delta_{0}+\mathrm{SUPEXP}$ is the extension of $E A$ by a $\Pi_{2}$ axiom asserting the totality of superexponentiation function $2_{x}^{y}$ (cf [3]). A theory $T$ is $\Pi_{n}$ axiomatized, if all of its nonlogical axioms are $\Pi_{n}$ sentences.

Theorem 1. Let $T$ be an arithmetical theory containing EA. Then, for any $n \geq 2,\left[T, \Pi_{n}-\mathrm{IR}\right]$ is equivalent to $T$ together with $\mathrm{RFN}_{\Pi_{n}}\left(T_{0}\right)$ for all finite $\Pi_{n+1}$ axiomatized subtheories $T_{0}$ of $T$. This statement also holds for $n=1$, provided $T$ contains $I \Delta_{0}+\mathrm{SUPEXP}$.

Our proof of Theorem 1 is based upon quite standard techniques that combines Tarski's method of partial truthdefinitions with the formalization of the Cut-elimination Theorem, and is, in fact, very close to the proof of Leivant's theorem (cf [7]). The proof admits an easy direct argument, without any use of skolemization. We need a few standard prerequisites.

Sequent calculus. We adopt a variant of the sequent calculus from [17], i.e., sequents are sets of formulas understood as big disjunctions, negations are treated via de Morgan's laws, etc.

Partial truth definitions. There is a $\Pi_{n}$ formula $\operatorname{True}_{\Pi_{n}}(x)$, which adequately expresses the predicate " $x$ is a Gödel number of a true $\Pi_{n}$ sentence" in $E A .{ }^{2}$ This

\footnotetext{
${ }^{2}$ We assume in this section that the class of $\Pi_{n}$ formulas contains not only those literally in $\Pi_{n}$ form, but also the ones obtained from prenex $\Pi_{n}$ formulas using $\vee, \wedge$, and universal quantification.
} 
means that $\operatorname{True}_{\Pi_{n}}(x)$ is well defined on atomic formulas and provably in $E A$ commutes with boolean connectives and quantifiers, i.e., satisfies Tarski conditions for $\Pi_{n}$ formulas. As a result, for any $A(x) \in \Pi_{n}$, we have:

$$
E A \vdash \forall x\left(A(x) \leftrightarrow \operatorname{True}_{\Pi_{n}}(\ulcorner A(\dot{x})\urcorner)\right) .
$$

For our proof it will be essential that Tarski conditions not only hold locally, for each individual $\Pi_{n}$ formula, but also uniformly so. In other words, $E A$ proves that, for all $\phi, \psi, \theta, \alpha, \gamma$ such that $\phi, \neg \phi, \psi, \theta, \forall x \gamma(x), \exists x \alpha(x)$ are $\Pi_{n}$ sentences,

$$
\begin{aligned}
\operatorname{True}_{\Pi_{n}}(\ulcorner\neg \phi\urcorner) & \leftrightarrow \operatorname{True}_{\Pi_{n}}(\ulcorner\phi\urcorner), \\
\operatorname{True}_{\Pi_{n}}(\ulcorner\theta \wedge \psi\urcorner) & \leftrightarrow \operatorname{True}_{\Pi_{n}}(\ulcorner\theta\urcorner) \wedge \operatorname{True}_{\Pi_{n}}(\ulcorner\psi\urcorner), \\
\operatorname{True}_{\Pi_{n}}(\ulcorner\theta \vee \psi\urcorner) & \leftrightarrow \operatorname{True}_{\Pi_{n}}(\ulcorner\theta\urcorner) \vee \operatorname{True}_{\Pi_{n}}(\ulcorner\psi\urcorner), \\
\operatorname{True}_{\Pi_{n}}(\ulcorner\exists x \alpha(x)\urcorner) & \leftrightarrow \exists x \operatorname{True}_{\Pi_{n}}(\ulcorner\alpha(\dot{x})\urcorner), \\
\operatorname{True}_{\Pi_{n}}(\ulcorner\forall x \gamma(x)\urcorner) & \leftrightarrow \forall x \operatorname{True}_{\Pi_{n}}(\ulcorner\gamma(\dot{x})\urcorner) .
\end{aligned}
$$

Let me stress that $\phi, \psi, \ldots$ here are variables over Gödel numbers of sentences, rather than individual sentences. (The standard dots-and-corners notation is somewhat sloppy in this respect. Yet, we hope that this will not create serious problems for the reader.)

On a par with the definition of truth, we also have a reasonable evaluation of terms in $E A$, that is, a definable Kalmar elementary function eval $(u, x)$ which provably commutes with $0,{ }^{\prime},+, \cdot$ and therefore, for any term $t\left(x_{1}, \ldots, x_{n}\right)$, satisfies

$$
E A \vdash \forall x_{1}, \ldots, x_{n} \operatorname{eval}\left(\ulcorner t\urcorner,\left\langle x_{1}, \ldots, x_{n}\right\rangle\right)=t\left(x_{1}, \ldots, x_{n}\right) .
$$

Usually, eval $(u, x)$ is explicitly used in the construction of a truthdefinition for the evaluation of atomic formulas. This implies that the truthdefinition and the evaluation of terms agree in the sense that, provably in $E A$, for all $\Pi_{n}$ formulas $\phi(a)$ and terms $t(a)$

$$
\forall x, y\left[\operatorname{eval}(t,\langle x\rangle)=y \rightarrow\left(\operatorname{True}_{\Pi_{n}}(\ulcorner\phi(t(\dot{x}))\urcorner) \leftrightarrow \operatorname{True}_{\Pi_{n}}(\ulcorner\phi(\dot{y})\urcorner)\right)\right],
$$

and similarly for terms $t$ and formulas $\phi$ in more than one free variable.

Now let $T$ be a finitely axiomatized theory, and let $\neg T$ denote the negation of the conjuction of all axioms of $T$. Furthermore, let $I_{T}(m)$ denote the formula expressing the following:

"For all $p$, if $p$ is a cut-free derivation of a sequent of the form $\neg T, \Pi(a)$, where $\Pi(a)$ contains $\Pi_{n}$ formulas only and $a$ stands for all the free variables in $\Pi$, and if the height of $p$ is $<m$, then $\forall x \operatorname{True}_{\Pi_{n}}(\ulcorner\bigvee \Pi(\dot{x})\urcorner)$."

Clearly, $I_{T}(m)$ is a $\Pi_{n}$ formula. 
Lemma 3.1. Suppose $T$ is a finite $\Pi_{n+1}$ axiomatized extension of EA. Then

$$
T \vdash I_{T}(0) \wedge \forall m\left(I_{T}(m) \rightarrow I_{T}(m+1)\right) .
$$

Proof. We reason informally within $T$. We may assume that the single nonlogical axiom of $T$ has the form $\forall z \neg \alpha(z)$, where $\alpha(z)$ is a $\Pi_{n}$ formula with $n$ alternating leading quantifiers followed by a bounded formula. $I_{T}(0)$ trivially holds. We show that $I_{T}(m)$ implies $I_{T}(m+1)$. Thus, we are given a cut-free derivation, of height $m+1$, of a sequent of the form $\exists z \alpha(z), \Pi$ for a finite set of $\Pi_{n}$ formulas $\Pi$. We must show that the disjunction of $\Pi$ is True, in the sense of True $_{\Pi_{n}}$, under every substitution of numerals for free variables in $\Pi$. For the rest of the proof we fix an arbitrary substitution of this kind and treat $\Pi$ as if it were a set of sentences. We distinguish several cases, according to the form of the last rule applied in the given derivation.

CASE 1. The sequent $\exists z \alpha(z), \Pi$ is a logical axiom, that is, has the form $\Gamma, \phi, \neg \phi$ for some $\phi$. Since $\exists z \alpha(z)$ is the only formula of the sequent that has complexity higher than $\Pi_{n}$, both $\phi$ and $\neg \phi$ must belong to $\Pi$. Tarski commutation conditions then imply that

$$
\operatorname{True}_{\Pi_{n}}(\ulcorner\neg \phi\urcorner) \leftrightarrow \neg \operatorname{True}_{\Pi_{n}}(\ulcorner\phi\urcorner) .
$$

So we obtain $\operatorname{True}_{\Pi_{n}}(\ulcorner\phi\urcorner) \vee \operatorname{True}_{\Pi_{n}}(\ulcorner\neg \phi\urcorner)$ and hence $\operatorname{True}_{\Pi_{n}}(\ulcorner\bigvee \Pi\urcorner)$.

CASE 2. The sequent $\exists z \alpha(z), \Pi$ is obtained by a rule introducing a logical connective into a formula from $\Pi$. All these rules are treated similarly using the subformula property of cut-free derivations and Tarski commutation conditions for True $_{\Pi_{n}}$. For example, the rule for the universal quantifier has the form

$$
\frac{\exists z \alpha(z), \Pi^{\prime}, \phi(a)}{\exists z \alpha(z), \Pi^{\prime}, \forall x \phi(x)},
$$

where $a$ is not free in $\Pi^{\prime}$. We must show that the formula $\bigvee \Pi^{\prime} \vee \forall x \phi(x)$ is True. By the induction hypothesis, since $a$ does not occur free in $\Pi^{\prime}$, we know that, for each $x, \bigvee \Pi^{\prime} \vee \phi(\bar{x})$ is True. Commuting True $\Pi_{n}$ with the small disjunction we conclude that, for each $x$, either $\bigvee \Pi^{\prime}$ or $\phi(\bar{x})$ is True. Since $\Pi^{\prime}$, and also True $\Pi_{n}\left(\left\ulcorner\Pi^{\prime}\right\urcorner\right)$, do not depend on $x$, it follows that either $\Pi^{\prime}$ is True, or for every $x, \phi(\bar{x})$ is True. Commuting True $\Pi_{n}$ with the universal quantifier and then backwards with the disjunction we conclude that $\Pi^{\prime} \vee \forall x \phi(x)$ is True, q.e.d.

In the remaining case we shall be more explicit about parameters.

CASE 3. The last rule introduces the existential quantifier in front of $\alpha(z)$, i.e., our derivation has the form

$$
\frac{(\exists z \alpha(z)), \alpha(t(a)), \Pi(a)}{\exists z \alpha(z), \Pi(a)} .
$$

A free variable $a$ here stands for all the parameters on which $\Pi$ and the term $t$ may depend. Extra parentheses indicate that the formula $\exists z \alpha(z)$ in the premise 
may occur as well as not. Without loss of generality we may assume that it does occur. (Otherwise apply Weakening Lemma 2.3.1 from [17] in order to obtain a derivation of the same height where the formula in question occurs.)

So, the induction hypothesis is applicable and implies that, for all $x$, either the disjunction of $\Pi(\bar{x})$, or $\alpha(t(\bar{x}))$ is True. We must, reasoning inside $T$, refute the second alternative.

Notice that, although, in general, $t$ is a "nonstandard" term, $\alpha(z)$ is a fixed "standard" $\Pi_{n+1}$ formula. Therefore Tarski's commutation lemma (14) can be applied to $\alpha(z)$, after evaluating the term $t$. Thus, by (15) and (14) we obtain:

$$
\begin{aligned}
\operatorname{True}_{\Pi_{n}}(\ulcorner\alpha(t(\dot{x}))\urcorner) \wedge \operatorname{eval}(t,\langle x\rangle)=y & \rightarrow \operatorname{True}_{\Pi_{n}}(\ulcorner\alpha(\dot{y})\urcorner) \\
& \rightarrow \alpha(y)
\end{aligned}
$$

Since the evaluation function is provably total in $E A$, it follows that $\operatorname{True}_{\Pi_{n}}(\ulcorner\alpha(t(\dot{x}))\urcorner)$ implies $\exists y \alpha(y)$, that is, yields a contradiction in $T$. Thus, we see that, for any $t$ and $x$, the formula $\alpha(t(\bar{x}))$ cannot be True, hence True is the disjunction of $\Pi(\bar{x})$, q.e.d.

\section{Proof of Theorem 1.}

1. $\left[T, \Pi_{n}\right.$-IR] is the theory axiomatized over $T$ by all formulas $\forall x I(x)$ such that $I(x) \in \Pi_{n}$ and $T$ proves

$$
I(0) \wedge \forall x(I(x) \rightarrow I(x+1)) .
$$

Therefore, first we must show that, for any such $I(x)$, there is a finite $\Pi_{n+1}$ axiomatized subtheory $T_{0} \subseteq T$ such that

$$
T+\operatorname{RFN}_{\Pi_{n}}\left(T_{0}\right) \vdash \forall x I(x) .
$$

For the axioms of $T_{0}$ we simply take $\Pi_{n+1}$ formula (16) together with all axioms of $E A$. Obviously, for every $n$ we have $T_{0} \vdash I(\bar{n})$. Furthermore, formalizing this fact in $E A$ we obtain:

$$
E A \vdash \forall x \operatorname{Prov}_{T_{0}}(\ulcorner I(\dot{x})\urcorner) .
$$

This implies $\forall x I(x)$ by $T_{0}$-reflection.

2. Now we must show that

$$
\left[T, \Pi_{n}-\mathrm{IR}\right] \vdash \mathrm{RFN}_{\Pi_{n}}\left(T_{0}\right),
$$

for any finite $\Pi_{n+1}$ axiomatized subtheory $T_{0} \subseteq T$. Let $I_{T_{0}}(m)$ be the formula defined in Lemma 3.1. Since $T_{0} \subseteq T$ and $I_{T_{0}}(m) \in \Pi_{n}$, we conclude that

$$
\left[T, \Pi_{n}-\mathrm{IR}\right] \vdash \forall m I_{T_{0}}(m) .
$$

Notice that for $T$ containing $E A$ and $n \geq 2$, obviously,

$$
\left[T, \Pi_{n}-\mathrm{IR}\right] \vdash \mathrm{SUPEXP} .
$$


On the other hand, it is well known (cf [3]) that $I \Delta_{0}+$ SUPEXP is a strong enough theory to prove the Cut-elimination Theorem for first order logic. In order to derive $\operatorname{RFN}_{\Pi_{n}}\left(T_{0}\right)$ we reason inside $\left[T, \Pi_{n}\right.$-IR], for every particular $\Pi_{n}$ formula $A(x)$, as follows.

Suppose $\operatorname{Prov}_{T}(\ulcorner A(\dot{x})\urcorner)$. Then the sequent $\neg T, A(\bar{x})$ is logically provable. By (formalized) Cut-elimination Theorem we obtain a cut-free proof of this sequent, and by (17) conclude that True $_{\Pi_{n}}(\ulcorner A(\dot{x})\urcorner)$ holds. Tarski commutation lemma (14) then yields $A(x)$, q.e.d.

The rest of this section is devoted to various remarks, corollaries and comments concerning Theorem 1 . Let, for a fixed $n \geq 1,(T)_{k}^{n}$ denote the sequence of theories based on iteration of the $\Pi_{n}$ reflection principle over $T$ :

$$
(T)_{0}^{n} \rightleftharpoons T, \quad(T)_{k+1}^{n} \rightleftharpoons(T)_{k}^{n}+\operatorname{RFN}_{\Pi_{n}}\left((T)_{k}^{n}\right), \quad(T)_{\omega}^{n} \rightleftharpoons \bigcup_{k \geq 0}(T)_{k}^{n}
$$

Similarly, $\left[T, \Pi_{n}-\mathrm{IR}\right]_{k}$ is defined by repeated application of $\Pi_{n^{-}}$IR:

$$
\left[T, \Pi_{n}-\mathrm{IR}\right]_{0} \rightleftharpoons T, \quad\left[T, \Pi_{n}-\mathrm{IR}\right]_{k+1} \rightleftharpoons\left[\left[T, \Pi_{n}-\mathrm{IR}\right]_{k}, \Pi_{n}-\mathrm{IR}\right] .
$$

We obviously have

$$
T+\Pi_{n}-\mathrm{IR} \equiv \bigcup_{k \geq 0}\left[T, \Pi_{n}-\mathrm{IR}\right]_{k} .
$$

Since for r.e. $T$ containing $E A$ the schema $\operatorname{RFN}_{\Pi_{n}}(T)$ is equivalent to a single $\Pi_{n}$ sentence, Theorem 1 can be applied repeatedly and we obtain

Corollary 3.1. Let $T$ be a finite $\Pi_{n+1}$ axiomatized theory containing $E A$ (or $I \Delta_{0}+\mathrm{SUPEXP}$ for $n=1$ ). Then

$$
T+\Pi_{n}-\mathrm{IR} \equiv(T)_{\omega}^{n}
$$

Moreover, for all $k \geq 1$, we actually have

$$
\left[T, \Pi_{n}-\mathrm{IR}\right]_{k} \equiv(T)_{k}^{n}
$$

that is, $k$ (nested) applications of induction rule precisely correspond to $k$ iterations of reflection principle over $T$.

Corollary 3.2. For theories $T$ as in the above corollary the closure of $T$ under $\Pi_{n}$ induction rule is a reflexive theory, and hence it is not finitely axiomatizable, unless it is inconsistent. The same holds for any extension of $T+\Pi_{n}$ IR by $\Sigma_{n}$ sentences. 
Remark 3.1. Theorem 1 shows that some conservation results for fragments of arithmetic and for iterated reflection principles are mutually interderivable. A well-known theorem due to Parsons, Mints, Takeuti and others states that $I \Sigma_{n}$ is conservative over $E A+\Sigma_{n}$-IR and $E A+\Pi_{n+1}$-IR for $\Pi_{n+1}$ sentences. This result follows at once from Leivant's equivalent characterization of $\Sigma_{n}$-IA as $\operatorname{RFN}_{\Pi_{n+2}}(E A)$ over $E A$ (cf [7]) and the characterization of $\Pi_{n+1}$-IR in terms of reflection principles in Corollary 3.1. Indeed, by the so-called Fine Structure Theorem of U.Schmerl (cf [15]) we know that $\operatorname{RFN}_{\Pi_{n+2}}(E A)$ is $\Pi_{n+1}$ conservative over $(E A)_{\omega}^{n+1}$, which is equivalent to $E A+\Pi_{n+1}$-IR by Corollary $3.1 .^{3}$ On the other hand, this particular case of Schmerl's theorem obviously follows from Parsons' result, too. The relationship between the 4 mentioned results can be summarized in the following diagram:

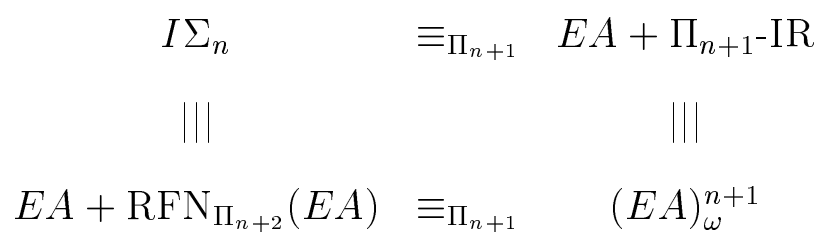

The 'horizontal' conservation results are due to Parsons and Schmerl, and the 'vertical' equivalences are Leivant's and ours (Corollary 3.1).

Remark 3.2. An interesting particular case of Theorem 1 concerns the induction rule for $\Pi_{1}$ formulas. It is well-known that the uniform reflection principle for $\Pi_{1}$ formulas for a theory $T$ is equivalent to consistency assertion for $T$, Con $(T)$. So, Corollary 3.1 can be reformulated as follows: for finite $\Pi_{2}$ axiomatized theories $T$ containing $I \Delta_{0}+$ SUPEXP,

$$
T+\Pi_{1}-\mathrm{IR} \equiv T+\operatorname{Con}(T)+\operatorname{Con}(\operatorname{Con}(T))+\ldots
$$

Clearly, for a sound theory $T, T+\Pi_{1}$-IR is an extension of $T$ by true $\Pi_{1}$ axioms, and hence both $T$ and $T+\Pi_{1}$-IR have the same class of provably recursive functions. Despite that, $T+\Pi_{1}-\mathrm{IR}$ is stronger than $T$ and the equivalence (18) gives us a precise measure of its relative strength.

Remark 3.3. In paper [19] there is a confusion concerning $\Pi_{1}-\mathrm{IR}$. Theorem 2.1.3 of that paper is false for it implies that $E A+\Pi_{1}$-IR contains more provably recursive functions than $E A$. (Lemma 2.1.4 is true, but it is not difficult to see that the schema of 'restricted primitive recursion' dealt with there is actually equivalent to the unrestricted primitive recursion. So, the proof-theoretic analysis in this lemma gives us no more information about the strength of $\Pi_{1}$-IR than the reduction of $\Pi_{1}$-IR to $\Sigma_{1}$-IR.)

\footnotetext{
${ }^{3}$ Schmerl formulated his result for the hierarchy of (transfinitely iterated) reflection principles over PRA, but it is not difficult to check that his proof essentially works over $E A$ as well.
} 
Theorem 2.1.7 of that paper states that the closure of $E A$ under $k$ applications of $\Pi_{2}$-IR (in our terminology, $\left[E A, \Pi_{2}-\mathrm{IR}\right]_{k}$ ) is conservative over the arithmetic corresponding to the $(k+3)$-d class of Grzegorczyk hierarchy. This theorem is correct and closely parallel to another particular case of Theorem 3.1. In fact, Theorem 2.1.7 also follows from our results in Section 7.

Remark 3.4. A characterization of $\Pi_{1}$-IR for theories weaker than $I \Delta_{0}+$ SUPEXP can be obtained in the spirit of Wilkie and Paris [22]. In this situation the family of consistency assertions w.r.t. proofs of bounded cut-rank $\operatorname{Con}_{k}(T), k \geq 0$, plays the role of the single consistency assertion $\operatorname{Con}(T)$ for $T$. Since $E A$ is a strong enough theory to prove Cut-elimination Theorem for derivations of bounded cutrank, a quick inspection of the given proof of Theorem 1 yields the following result: for $T$ containing $E A,\left[T, \Pi_{1}\right.$-IR] is equivalent to $T$ together with all $\mathrm{Con}_{k}(U)$ such that $k \geq 0$ and $U$ is a finite $\Pi_{2}$ axiomatized subtheory of $T$.

Our next goal is the characterization of $\Sigma_{n}$ induction rule in the spirit of Theorem 1. Parsons showed that $\Sigma_{n}$-IR is equivalent to $\Pi_{n+1}$-IR over $E A$. However, the two rules are not congruent and so, a more careful analysis is needed here. Let me explain why the simple proof of Theorem 1 cannot be easily adapted to the $\Sigma_{n}$ case.

The technical reason is that the formula $I_{T}(m)$ in that proof involves a number of outer universal quantifiers, and therefore does not have the reqired $\Sigma_{n}$ form. Some of these quantifiers, e.g., the quantifier over all derivations $p$, can actually be bounded. One can replace the induction on the height $m$ of a proof by $\mathrm{IR}_{<}$ over Gödel numbers $p$ of proofs using the fact that, under the standard coding, subderivations of $p$ have smaller Gödel numbers. However, there does not seem to be an easy way to get rid of the quantifier over all substitutions of numerals for free variables in the end-sequent. The only possibility here seems to be to keep those variables free, as the parameters of the formula $I_{T}$. Yet, this possibility is blocked by the simple fact that, for large $n$, some sequents in the proof $p$ may contain many more parameters than the end-sequent, and we ought to take them all into account. There is one rare situation where this difficulty does not arise: simply, if there are no universal quantifiers in the end-sequent. This idea allows us to analyse the $\Sigma_{1}$ induction rule. Then, by skolemization, we will be able to pull the result up in the arithmetical hierarchy. This project is carefully elaborated in the remaining part of the paper.

\section{Provably recursive functions}

In this section we recall some basic facts about provably (total) recursive functions (p.t.r.f.s) of theories and characterize these functions for closures of theories under $\Sigma_{1}$ induction rule. Most of this material is fairly standard, so we are sketchy about proofs. 
We shall deal with various classes of number-theoretic functions. The basic class is the class of elementary functions $\mathcal{E}$. For a class $K, \mathbf{C}(K)$ denotes the closure of $K \cup \mathcal{E}$ under composition. $[K, \mathbf{P R}]$ denotes the closure of $K$ under composition and one application of primitive recursion, i.e., the class $\mathbf{C}(F)$, where $F$ is the set of all functions $f(n, a)$ definable by a schema of the form

$$
\left\{\begin{aligned}
f(0, a) & =g(a) \\
f(n+1, a) & =h(f(n, a), n, a)
\end{aligned}\right.
$$

for $g, h \in \mathbf{C}(K) . \quad \mathbf{E}(K)$ is the elementary closure of $K$, that is, the class of functions obtained from $K \cup \mathcal{E}$ by composition and bounded sums and products.

Definition 4. A number-theoretic function $f(x)$ is called provably recursive in a theory $T$ iff the graph of $f$ can be represented by a $\Sigma_{1}$ formula $\psi(x, y)$ such that

$$
T \vdash \forall x \exists ! y \psi(x, y) \text {. }
$$

The class of p.t.r.f.s of a theory $T$ is denoted $\mathcal{D}(T)$.

It is easy to see that graphs of p.t.r.f.s are actually $\Delta_{1}$ in $T$. The class $\mathcal{D}(T)$ is closed under composition, but not necessarily elementarily closed, even if $T$ contains $E A$. This creates for us some additional difficulties, since proof-theoretically it is much more common and pleasant to deal with elementarily closed classes of functions. Sometimes one considers p.t.r.f.s with elementary graphs in place of $\Sigma_{1}$ graphs. These classes of functions are closed under bounded recursion (sums and products), but not under composition. However, the following obvious proposition holds.

Proposition 4.1. For a theory $T$ containing EA, every p.t.r.f. can be obtained by composition from a p.t.r.f. with an elementary graph and a fixed elementary function.

Proof. Let $\psi(x, y):=\exists z \psi_{0}(z, x, y)$, where $\psi_{0}$ is elementary, define the graph of $f$, so that

$$
T \vdash \forall x \exists ! y \psi(x, y) .
$$

Using the standard pairing function we let

$$
\phi(x, y):=\psi_{0}\left((y)_{0}, x,(y)_{1}\right) \wedge \forall z<(y)_{0} \neg \psi_{0}\left(z, x,(y)_{1}\right) \text {. }
$$

Then it is not difficult to check that $\phi$ defines a certain p.t.r.f. in $T, \phi$ is elementary, and for all $n, f(n)=(g(n))_{1}$, q.e.d.

Since $\mathcal{D}(T)$ only depends on the $\Pi_{2}$ fragment of $T$, we shall concentrate our attention on $\Pi_{2}$ axiomatized theories. 
Definition 5. Let $\pi:=\forall x \exists y \phi(x, y) \in \Pi_{2}$, with $\phi$ elementary. A function $f(x)$ is called a witness of $\pi$ iff $\forall x \phi(x, f(x))$ holds in the standard model of arithmetic.

Every true $\Pi_{2}$ sentence has a witness. The function $f_{\pi}(x)$ whose graph is defined by the formula $\phi(x, y) \wedge \forall z<y \neg \phi(x, z)$ is called the standard witness of $\pi$.

Proposition 4.2. Let $T$ be a finite $\Pi_{2}$ axiomatized sound extension of $E A$, and let $f$ be the standard witness of the single axiom of $T$. Then $\mathcal{D}(T)=\mathbf{C}(f)$.

Proof. Obviously, $f$ is a p.t.r.f. in $T$, and so $\mathbf{C}(f) \subseteq \mathcal{D}(T)$. The opposite inclusion is, more or less, a direct consequence of Herbrand's Theorem. Consider a purely universal formulation of $E A$ (in a language with symbols for all Kalmar elementary functions), and add to this language a new function symbol $f$ together with the axiom

$$
\forall x \phi(x, f(x)),
$$

where $\forall x \exists y \phi(x, y)$ is the single axiom of $T$. Using appropriate Kalmar elementary terms we can get rid of all bounded quantifiers in $\phi$. Hence the resulting theory is a conservative extension of $T$ and has a purely universal axiomatization.

Now suppose $T \vdash \forall x \exists ! y \exists z \psi_{0}(x, y, z)$, where $\psi_{0}$ is elementary (and in our formulation also quantifier-free). Since $T$ has a purely universal axiomatization, by Herbrand's Theorem we obtain terms $t_{1}, \ldots, t_{k}, u_{1}, \ldots, u_{k}$ of the extended language such that

$$
T \vdash \psi_{0}\left(a, t_{1}(a), u_{1}(a)\right) \vee \ldots \vee \psi_{0}\left(a, t_{k}(a), u_{k}(a)\right) .
$$

Clearly, the terms $t_{i}$ and $u_{i}$ represent functions in $\mathbf{C}(f)$. Now we let

$$
t(x):= \begin{cases}t_{1}(x), & \text { if } \psi_{0}\left(x, t_{1}(x), u_{1}(x)\right), \\ t_{2}(x), & \text { if } \psi_{0}\left(x, t_{2}(x), u_{2}(x)\right) \text { and } \neg \psi_{0}\left(x, t_{1}(x), u_{1}(x)\right), \\ \cdots & \cdots \\ t_{k}(x), & \text { if } \psi_{0}\left(x, t_{k}(x), u_{k}(x)\right) \text { and } \neg \psi_{0}\left(x, t_{i}(x), u_{i}(x)\right) \text { for all } i<k, \\ 0, & \text { otherwise. }\end{cases}
$$

The function $u(x)$ is defined in a similar manner, with $u_{i}$ 's in place of $t_{i}$ 's. Since the function

$$
\operatorname{Cond}(x, y, z):= \begin{cases}x, & \text { if } z=0 \\ y, & \text { if } z \neq 0\end{cases}
$$

is elementary, the class $\mathbf{C}(f)$ is closed under definitions by cases and so, $t(x)$ and $u(x)$ can be adequately defined by $\mathbf{C}(f)$ terms. For these terms we obviously have $T \vdash \psi_{0}(a, t(a), u(a))$. It follows that

$$
T \vdash \forall x \exists z \psi_{0}(x, t(x), z),
$$

and by the functionality of $\psi$

$$
T \vdash \forall x, y(t(x)=y \leftrightarrow \psi(x, y)) .
$$


Since all theorems of $T$ are true, $\psi$ represents the graph of $t(x)$ in the standard model, q.e.d.

Remark 4.1. We have actually shown that $\mathcal{D}(T) \subseteq \mathbf{C}(f)$ for any witness $f$ of the axiom of $T$, not just for the standard one.

Corollary 4.1. Let $T$ be a finite $\Pi_{2}$ axiomatized sound extension of $E A$. Then the class $\mathcal{D}(T)$ has a finite basis under composition.

Proof: Follows from the previous proposition and the fact that $\mathcal{E}$ has a finite basis (cf, e.g., $[8,10])$. It might be interesting for the reader to notice that, if we had been slightly more careful in the proof of Proposition 4.2, we could actually have inferred the existence of a finite basis in $\mathcal{E}$ from finite axiomatizability of $E A$.

Consider a finite $\Pi_{2}$ axiomatization of $E A$ in the usual language of arithmetic (see [3]). Introduce finitely many (Kalmar elementary) functions to quantifierfree represent $\Delta_{0}$ parts of those $\Pi_{2}$ axioms. Then we have to introduce finitely many Skolem functions for these axioms in order to obtain a purely universal conservative extension of $E A$. Essentially the same proof as for Proposition 4.2 then shows that every provably recursive function can be defined by a term in the extended language. In the process we would have to introduce a few more functions like Cond $(x, y, z)$ or pairing functions. We omit the details, q.e.d.

Remark 4.2. The converse of the previous corollary does not hold, essentially because of the difference between provably recursive functions and programs. For example the theory $(E A)_{\omega}^{1}$ extends $E A$ purely universally and therefore has the same, finitely based, class of p.t.r.f.s. Yet, this theory is not finitely axiomatizable.

Proposition 4.3. Let $T$ be a finite $\Pi_{2}$ axiomatized sound extension of $E A$, and let $f$ be the standard witness of the single axiom of $T$. Then

$$
\mathcal{D}\left(\left[T, \Sigma_{1}-\mathrm{IR}\right]\right)=[\mathbf{C}(f), \mathbf{P R}] .
$$

Proof. 1. Let $g(n, x)$ be defined by a schema of primitive recursion

$$
\left\{\begin{aligned}
g(0, x) & =e(x) \\
g(n+1, x) & =h(g(n, x), n, x)
\end{aligned}\right.
$$

such that $e, h \in \mathbf{C}(f)$. Since all functions in $\mathbf{C}(f)$ are p.t.r.f. in $T$, graphs of $e$ and $h$ are defined by $\Sigma_{1}$ formulas $E(x, y)$ and $H(z, n, x, y):=\exists v H_{0}(v, z, n, x, y)$, with $H_{0}$ elementary.

The graph of $g$ is most naturally defined (in the standard model) by the following formula (that uses elementary coding of sequences):

$$
g(n, x)=y: \leftrightarrow \exists s \in \operatorname{Seq}\left((s)_{0}=e(x) \wedge \forall i<n(s)_{i+1}=h\left((s)_{i}, i, x\right) \wedge(s)_{n}=y\right) .
$$


However, in absense of $\Sigma_{1}$ collection principle this formula may not be equivalent to a $\Sigma_{1}$ formula within $T$. We modify it as follows (a somewhat similar trick was employed earlier in the proof of Proposition 2.2): $g(n, x)=y: \leftrightarrow$

$$
\exists s, v \in \operatorname{Seq}\left(E\left(x,(s)_{0}\right) \wedge \forall i<n H_{0}\left((v)_{i},(s)_{i}, i, x,(s)_{i+1}\right) \wedge(s)_{n}=y\right) .
$$

This formula is obviously $\Sigma_{1}$, and now we shall show the totality of $g$ in $\left[T, \Sigma_{1}\right.$-IR]. Clearly, $T \vdash \exists y g(0, x)=y$, because $e(x)$ is provably total. In order to see that

$$
T \vdash \forall n(\exists y g(n, x)=y \rightarrow \exists y g(n+1, x)=y)
$$

we argue informally as follows. Suppose $g(n, x)=y$ and thus we are given two sequences $s$ and $v$ of length $n+1$ satisfying (19). We have to construct appropriate sequences of length $n+2$. Since the function $h$ is provably total, we can find a $z$ such that $h(y, n, x)=z$. Hence there is a $w$ such that $H_{0}(w, y, n, x, z)$ holds. Pick any such $w$ and add the element $z$ to the end of the sequence $s$, and $w$ to the end of $v$. The resulting sequences are as required. Applying $\Sigma_{1}$-IR we obtain:

$$
\left[T, \Sigma_{1}-\mathrm{IR}\right] \vdash \forall n, x \exists y g(n, x)=y .
$$

To prove the functionality of $g$ we reason as follows. Let $R(n, s, v, x, y)$ denote the elementary part of the formula (19), and suppose we have $R\left(n, s_{1}, v_{1}, x, y_{1}\right)$ and $R\left(n, s_{2}, v_{2}, x, y_{2}\right)$. We prove $\forall i \leq n\left(s_{1}\right)_{i}=\left(s_{2}\right)_{i}$ by induction on $i$ (with $n, s_{j}, v_{j}, x, y_{j}$ as free paramemters). Notice that the induction is elementary, although it is applied as a schema rather than as a rule here. Basis and induction step follow at once from the functionality of $e$ and $h$. So we obtain $\left(s_{1}\right)_{n}=\left(s_{2}\right)_{n}$, and therefore $y_{1}=y_{2}$. Notice that the argument for the functionality was actually carried out in $T$.

2. Now we shall show that p.t.r.f.s of $\left[T, \Sigma_{1}\right.$-IR] belong to $[\mathbf{C}(f), \mathbf{P R}]$. Since $\left[T, \Sigma_{1}-\mathrm{IR}\right]$ is a sound $\Pi_{2}$ axiomatized theory, it suffices to demonstrate that every formula obtained by an application of $\Sigma_{1}$-IR has a witnessing function in the class $[\mathbf{C}(f), \mathbf{P R}]$. (Here we actually apply Remark 4.1 rather than Proposition 4.2.)

Consider an arbitrary elementary formula $A(x, y, a)$ such that

$$
\begin{aligned}
& T \vdash \exists y A(0, y, a), \text { and } \\
& T \vdash \forall x(\exists y A(x, y, a) \rightarrow \exists y A(x+1, y, a)) .
\end{aligned}
$$

By Proposition 4.2 we obtain functions $e(a)$ and $h(y, x, a)$ in $\mathbf{C}(f)$ such that $e$ witnesses $\forall a \exists y A(0, y, a)$, and $h$ witnesses

$$
\forall a, x, y \exists z(A(x, y, a) \rightarrow A(x+1, z, a)) .
$$

Consider a primitive recursion

$$
\left\{\begin{aligned}
g(0, a) & =e(a) \\
g(x+1, a) & =h(g(x, a), x, a) .
\end{aligned}\right.
$$


Straightforward induction on $x$ then shows that $A(x, g(x, a), a)$ holds in the standard model for all $x$ and $a$. This means that $g(x, a)$ witnesses $\forall x, a \exists y A(x, y, a)$, q.e.d.

\section{Elementary closure}

As we have noted before, the class $\mathcal{D}(T)$ need not be elementarily closed even if the theory $T$ contains $E A$. In this section we shall investigate this question in more detail and formulate sufficient conditions for $\mathcal{D}(T)$ to be elementarily closed. Versions of the following two propositions can be found in [10] with more complicated proofs.

For a function $f(x)$, let $\bar{f}(n):=\langle f(0), \ldots, f(n)\rangle$.

Proposition 5.1. $\mathbf{E}(f)=\mathbf{C}(\bar{f})$.

Proof. Obviously $\bar{f} \in \mathbf{E}(f)$, so $\mathbf{C}(\bar{f}) \subseteq \mathbf{E}(f)$. For the opposite inclusion we prove that $\sum_{i \leq x} g(i, y) \in \mathbf{C}(\bar{f})$ if $g(x, y) \in \mathbf{C}(\bar{f})$. (Bounded products are treated similarly.)

Let $(z \uparrow n)$ denote the initial segment of a sequence $z$ of length $n+1$. This function is clearly Kalmar elementary. Since $g \in \mathbf{C}(\bar{f}), g$ can be considered as a term in a language with symbols for all elementary functions and a symbol for $\bar{f}$. We systematically replace all occurrences of subterms of the form $\bar{f}(t)$ in $g$ by $(z\lceil t)$, where $z$ is a new variable. (It does not matter, in what order these occurrences are replaced.) As a result we obtain an elementary function $\tilde{g}(x, y, z)$. Define:

$$
G(x, y, z):=\sum_{i \leq x} \tilde{g}(i, y, z)
$$

We claim that

$$
\sum_{i \leq x} g(i, y)=G(x, y, \bar{f}(b(x, y))),
$$

for a certain term $b(x, y) \in \mathbf{C}(\bar{f})$. We only need to ensure that the value of $b(x, y)$ is greater than all values $t(i, y)$ for $i \leq x$, where terms $t$ occur in the context $\bar{f}(t)$ within $g$. Notice that $\bar{f}$ is an increasing function. Therefore we can majorize each $t(x, y)$ by an increasing function in $\mathbf{C}(\bar{f})$ and take the sum of all these functions as $b(x, y)$, q.e.d.

Proposition 5.2. If $f(x)$ is increasing and the graph of $f$ is elementary, then $\bar{f} \in \mathbf{C}(f)$ and therefore $\mathbf{C}(f)=\mathbf{E}(f)$.

Proof. If $f$ is increasing, for a certain elementary function $b$ we have

$$
\bar{f}(n)=\mu z \leq b(n, f(n)) . \forall i \leq n(z)_{i}=f(i),
$$

because the code of a sequence can be estimated elementarily in its length and the largest element $(=f(n))$, q.e.d. 
Definition 6. Let $\pi$ be a $\Pi_{2}$ sentence. $\pi$ is monotonic, if there is an elementary formula $\phi(x, y)$ such that $E A$ proves that

1. $\pi \leftrightarrow \forall x \exists y \phi(x, y)$,

2. $\phi(x, y) \wedge \phi(x, z) \rightarrow y=z$,

3. $\phi\left(x_{1}, y\right) \wedge \phi\left(x_{2}, z\right) \wedge x_{1} \leq x_{2} \rightarrow y \leq z$.

In other words, $\pi$ is monotonic iff it is equivalent to a sentence whose standard witness is provably increasing.

Proposition 5.3. Let $T$ be a $\Pi_{2}$ axiomatized theory containing EA. The following statements are equivalent:

1. $T$ is axiomatizable over $E A$ by monotonic $\Pi_{2}$ sentences;

2. $T$ is closed under $\Sigma_{1}$ collection rule:

$\Sigma_{1}$-CR: $\quad \forall x \exists y \phi(x, y) \vdash \forall x \exists y \forall u \leq x \exists v \leq y \phi(u, v)$,

where $\phi(x, y) \in \Sigma_{1}$.

Proof. Clearly, the formula $\forall x \exists y \forall u \leq x \exists v \leq y \phi(u, v)$ implies $\forall x \exists y \phi(x, y)$ in $E A$ and is monotonic, whenever $\phi$ is elementary. So, we may apply $\Sigma_{1}$ collection rule to all axioms of $T$ and obtain a monotonic axiomatization.

In order to show that Statement 1 implies 2 we take an axiomatization of $T$ over $E A$ by $\Pi_{2}$ formulas whose standard witnesses are monotonic. Then we introduce Skolem functions for all these formulas and replace axioms $\pi:=\forall x \exists y \phi(x, y)$ of $T$ by their skolemizations $\forall x \phi\left(x, f_{\pi}(x)\right)$. The resulting theory $T^{*}$ proves monotonicity of all these functions $f_{\pi}$ :

$$
x_{1} \leq x_{2} \rightarrow f_{\pi}\left(x_{1}\right) \leq f_{\pi}\left(x_{2}\right) .
$$

Besides, it is conservative over $T$, and has a purely universal axiomatization (if $E A$ is taken in a universal formulation).

Now assume $T \vdash \forall x \exists y \psi(x, y)$ for a formula $\psi \in \Sigma_{1}$. By Herbrand's Theorem we can obtain a monotonic term $t(x)$ in the extended language such that

$$
T^{*} \vdash \forall x \exists y \leq t(x) \psi(x, y) .
$$

(This actually is a version of Parikh's Theorem for $T^{*}$ (cf [3],p. 272). Here we use the fact that every elementary function can be majorized by an increasing one, and hence any term in the extended language can.) Provable monotonicity of $t(x)$ then implies:

$$
T^{*} \vdash \forall x \exists y_{=t(x)} \forall u \leq x \exists v \leq y \psi(u, v) .
$$

The result follows by conservativity of $T^{*}$ over $T$, q.e.d. 
Corollary 5.1. $A \Pi_{2}$ sentence $\forall x \exists y \phi(x, y)$ is monotonic iff

$$
E A \vdash \forall x \exists y \phi(x, y) \rightarrow \forall x \exists y \forall u \leq x \exists v \leq y \phi(u, v) .
$$

Corollary 5.2. If a sound theory $T$ containing $E A$ is closed under $\Sigma_{1}$ collection rule, then $\mathcal{D}(T)$ is elementarily closed.

Proof. By Proposition 5.3 the $\Pi_{2}$ fragment of $T$ is axiomatizable by a set of monotonic $\Pi_{2}$ sentences $\Pi$. By Proposition 4.2 we know that

$$
\mathcal{D}(T)=\mathbf{C}\left(\left\{f_{\pi}: \pi \in \Pi\right\}\right) .
$$

All functions $f_{\pi}$ are increasing and have elementary graphs, so the result follows by Proposition 5.2, q.e.d.

The following proposition reveals a useful 'smoothening' property of $\Sigma_{1}$ induction rule.

Proposition 5.4. For any theory $T$ extending $E A,\left[T, \Sigma_{1}-\mathrm{IR}\right]$ is axiomatizable by monotonic $\Pi_{2}$ sentences over $T$. If $T$ itself is $\Pi_{2}$ axiomatized, $\left[T, \Sigma_{1}\right.$-IR] is axiomatizable by monotonic $\Pi_{2}$ sentences over $E A$.

Proof. Suppose $\phi(x, y)$ is elementary and

$$
\begin{aligned}
& T \vdash \exists y \phi(0, y, a), \text { and } \\
& T \vdash \forall x(\exists y \phi(x, y, a) \rightarrow \exists y \phi(x+1, y, a)) .
\end{aligned}
$$

Then it is easy to see that

$$
T \vdash \forall x, y \exists z(\forall u \leq x \exists v \leq y \phi(u, v, a) \rightarrow \forall u \leq x+1 \exists v \leq z \phi(u, v, a)) .
$$

Applying $\Sigma_{1}$-IR we obtain

$$
\forall x \exists y \forall u \leq x \exists v \leq y \phi(u, v, a) .
$$

This shows that $\left[T, \Sigma_{1}\right.$-IR $]$ is axiomatized by monotonic sentences over $T$. Moreover, a similar argument shows that for each theorem of $T$ of the form $\forall x \exists y \phi(x, y)$, with $\phi$ elementary, the formula

$$
\forall x \exists y \forall u \leq x \exists v \leq y \phi(u, v)
$$

is provable in $\left[T, \Sigma_{1}\right.$-IR]. (This essentially means that $\Sigma_{1}$-CR $\leq \Sigma_{1}$-IR.) So, if $T$ is $\Pi_{2}$ axiomatized, in an axiomatization of $\left[T, \Sigma_{1}\right.$-IR] the axioms of $T$ can also be replaced by monotonic sentences, q.e.d. 


\section{Evaluation}

The aim of this section is to show that the universal function for the class of p.t.r.f.s of a finite $\Pi_{2}$ axiomatized theory $T$ belongs to $[\mathcal{D}(T), \mathbf{P R}]$, and therefore can be represented in $\left[T, \Sigma_{1}-\mathrm{IR}\right]$. As a byproduct we obtain a new and very transparent proof of a theorem of R.Peter (cf [13] and also [14]) stating that so-called nested recursion on $\omega$ is reducible to primitive recursion.

Let $f(x)$ be a function. Every function of the class $\mathbf{C}(f)$ can be represented by a term in a language containing a function symbol for $f$ and finitely many function symbols for a certain basis in $\mathcal{E}$ (cf Proposition 4.1). We call these functions initial functions, and the terms of this language will be called $f$-terms. We fix a natural elementary Gödel numbering of $f$-terms.

The evaluation function eval $_{f}(e, x)$ for $f$-terms is defined as follows:

$$
\operatorname{eval}_{f}(e, x):= \begin{cases}t\left((x)_{0}, \ldots,(x)_{n}\right), & \text { if } e=\ulcorner t\urcorner \text { for an } f \text {-term } t \text { of arity } n+1, \\ 0, & \text { otherwise. }\end{cases}
$$

\section{Proposition 6.1. $[\mathbf{C}(f), \mathbf{P R}]=\mathbf{C}\left(\mathrm{eval}_{f}\right)$}

Proof. First we show that eval $f$ belongs to $[\mathbf{C}(f), \mathbf{P R}]$. The definition of eval $f$ can obviously be rewritten as a primitive 'course of values' recursion:

1. $\operatorname{eval}_{f}(e, x):=g\left((x)_{0}, \ldots,(x)_{n}\right)$, if $e=\ulcorner g\urcorner$, and $g$ is an initial function,

2. $\operatorname{eval}_{f}(e, x):=h\left(\operatorname{eval}_{f}\left(\left\ulcorner t_{0}\right\urcorner, x\right), \ldots, \operatorname{eval}_{f}\left(\left\ulcorner t_{m}\right\urcorner, x\right)\right)$, if $e=\left\ulcorner h\left(t_{0}, \ldots, t_{m}\right)\right\urcorner$, and $h$ is an initial function,

3. eval $_{f}(e, x):=0$, if none of the above cases holds.

Since there are only finitely many initial functions, this definition has the form of a definition by cases. The cases are Kalmar elementarily recognizable by the naturality assumption on the coding of $f$-terms. Further, it is well known and easy to see that the 'course of values' recursion defining eval $f$ can be reduced to the usual primitive recursion for the function

$$
\overline{\operatorname{eval}}_{f}(e, x):=\left\langle\operatorname{eval}_{f}(0, x), \ldots, \operatorname{eval}_{f}(e, x)\right\rangle \text {, }
$$

from which eval $_{f}$ can be recovered as

$$
\operatorname{eval}_{f}(e, x)=\left(\overline{\operatorname{eval}}_{f}(e, x)\right)_{e}
$$

Now we shall show that $\mathbf{C}\left(\mathrm{eval}_{f}\right)$ contains $[\mathbf{C}(f), \mathbf{P R}]$. Consider a primitive recursive definition

$$
\left\{\begin{aligned}
g(0, a) & =g_{0}(a) \\
g(n+1, a) & =h(g(n, a), n, a)
\end{aligned}\right.
$$


for some $f$-terms $g_{0}(a)$ and $h(x, y, a)$. We shall express $g(n, a)$ in the form

$$
\operatorname{eval}_{f}(s(n),\langle a\rangle)
$$

for a function $s(n)$ to be found. Let num $(n)$ denote the index of a constant $f$-term with value $n$, and let $\operatorname{Sub}_{x y}(e, i, j)$ compute the index of an $f$-term that results in substitution of $f$-terms $i$ and $j$ for variables $x$ and $y$ respectively in an $f$-term $e$. It is easy to see that functions Sub and num are elementary. Then we can define $s(n)$ as follows:

$$
\left\{\begin{aligned}
s(0) & :=\left\ulcorner g_{0}\right\urcorner \\
s(n+1) & :=\operatorname{Sub}_{x y}(\ulcorner h\urcorner, s(n), \operatorname{num}(n)) .
\end{aligned}\right.
$$

It follows that

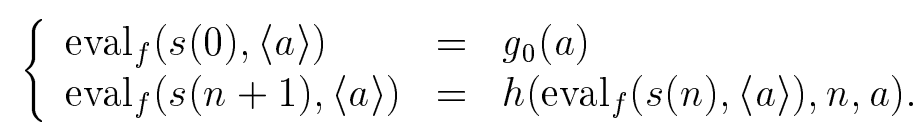

So, it only remains to prove that primitive recursion (20) is bounded. Let $|t|$ denote the length (= number of symbols) of a term with index $t$. For Sub we have the following estimate:

$$
\left|\operatorname{Sub}_{x y}(e, i, j)\right| \leq C \cdot|e| \cdot \max (|i|,|j|),
$$

because the total number of occurrences of variables $x$ and $y$ in a term $e$ is less than $|e|$. On the other hand, the length of num $(n)$ is at worst linear in $n$. So, for large enough $n$ we have:

$$
|s(n+1)| \leq C_{1} \cdot|s(n)|
$$

It follows that $|s(n)|$ grows at most exponentially, and thereby $s(n)$ has a doubly exponential bound, q.e.d.

Two immediate consequences of the above proposition are:

Corollary 6.1. The class $[\mathbf{C}(f), \mathbf{P R}]$ is finitely based.

Corollary 6.2. The class $\mathbf{C}\left(\mathrm{eval}_{f}\right)$ is elementarily closed.

Another interesting corollary is the reduction of nested recursion to primitive recursion. A nested recursive definition may have, e.g., the following form:

$$
\left\{\begin{aligned}
g(0, a) & =g_{0}(a) \\
g(n+1, a) & =h_{0}\left(g\left(n, h_{1}(g(n, a), a)\right), n, a\right) .
\end{aligned}\right.
$$

In general one allows arbitrarily deep nestings of $g$-terms on the right hand side of the definition, but $g$ must only occur in the context $g(n, \cdot)$, that is, the first 
argument must always be $n$. An old result of R.Peter says that nested recursion is reducible to primitive recursion, and it is relevant for our work as follows.

Suppose we want to evaluate a term $t(u(x))$, where $t$ and $u$ are complex terms. Doing this in the most straightforward manner we must first evaluate $u$ and then $t$, that is,

$$
\operatorname{eval}_{f}(\ulcorner t \circ u\urcorner, x)=\operatorname{eval}_{f}\left(\ulcorner t\urcorner, \operatorname{eval}_{f}(\ulcorner u\urcorner,\langle x\rangle)\right) .
$$

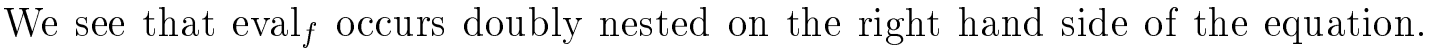
The evaluation procedure prescribed by Proposition 6.1 is different: we look at the terms $t$ and $u$ as being decomposed into initial functions, and evaluate only one function at a step. This is a longer process, although it yields the same result.

A natural rule to verify the totality of functions defined by nested recursion is $\Pi_{2}$ induction rule, rather than $\Sigma_{1}$-IR, which only works for primitive recursive definitions on the face of it. ${ }^{4}$ Therefore it is not surprising that Peter's theorem is an essential element in Parsons' proof of the equivalence of $\Pi_{2}$ and $\Sigma_{1}$ induction rules. Here we obtain a slightly sharpened version of Peter's result for free.

Corollary 6.3. The closure of a class $K$ of functions containing $\mathcal{E}$ under one application of nested recursion and composition coincides with $[K, \mathbf{P R}]$.

Proof. Without loss of generality we may assume that $K$ has the form $\mathbf{C}(f)$. Now we almost literally follow the lines of the proof of the second part of Proposition 6.1. A function $g(n, a)$ defined by nested recursion from $\mathbf{C}(f)$ can be expressed in the form $\operatorname{eval}_{f}(s(n), a)$ for a suitable elementary function $s$. The bound on the rate of growth of $s$, however, will be slightly worse than before. For sufficiently large $n$ we have

$$
|s(n+1)| \leq C \cdot|s(n)|^{k},
$$

where $k$ is the maximum depth of nestings in the definition of $g$. However, this means that $s$ grows no faster than triply exponentially, q.e.d.

Let $T$ be finite $\Pi_{2}$ axiomatized extension of $E A$ and let $f$ be the standard witness for the single axiom of $T$. We shall show that the evaluation function for $f$-terms can be naturally represented in $\left[T, \Sigma_{1}\right.$-IR], and that its basic properties are provable in this theory. Essentially, the argument is a careful inspection of the proofs of Propositions 4.3 and 6.1, but there are a few difficulties that we must explain how to avoid.

Without loss of generality we may assume that $T$ is formulated in a language containing function symbols for $f$ and for finitely many initial elementary functions. (However, when speaking about the complexity of formulas we shall always

\footnotetext{
${ }^{4}$ A recently introduced 'Logic of Primitive Recursion' by Sieg and Wainer [20] seems to provide a relevant framework for the analysis of the intensional phenomenon of correpondence between rules and computational schemes.
} 
refer to their translations into the standard language of arithmetic. Recall that the graph of $f$ is defined by an elementary formula.)

By Propositions 4.3 and 6.1 we know that eval $f$ is provably recursive in $\left[T, \Sigma_{1}\right.$-IR], and hence its graph can be represented by a certain $\Sigma_{1}$ formula. This formula can be read off from the primitive recursive definition of eval $f$, or rather $\overline{\mathrm{eval}}_{f}$, given in the proof of Proposition 6.1 and using the formalization of primitive recursion (19) in the proof of Proposition 4.3. All our considerations below will concern this particular formula.

Let $\mathrm{EVAL}_{f}$ denote the $\Pi_{2}$ formula expressing the totality of eval $\mathrm{f}_{\mathrm{f}}$

$$
\mathrm{EVAL}_{f}:=\forall e, x \exists y \operatorname{eval}_{f}(e, x)=y \text {. }
$$

First of all, we know that $\left[T, \Sigma_{1}\right.$-IR] proves EVAL $_{f}$. Besides, since the formula $\mathrm{EVAL}_{f}$ is read off from the primitive recursive definition of eval $f$, recursive Clauses $1-3$ out of the proof of Proposition 6.1 are provable in $T$. That is, for all initial functions $g\left(x_{0}, \ldots, x_{n}\right)$ and $h\left(x_{0}, \ldots, x_{m}\right)$ we have:

$$
\begin{aligned}
& T \vdash \forall x, y, e\left(\operatorname{eval}_{f}(e, x)=y \wedge e=\ulcorner g\urcorner \rightarrow y=g\left((x)_{0}, \ldots,(x)_{n}\right)\right), \\
& T \vdash \forall e, x, e_{0}, \ldots, e_{m}, y, y_{0}, \ldots, y_{m}\left[e=\operatorname{Sub}_{x_{0} \cdots x_{m}}\left(\ulcorner h\urcorner, e_{0}, \ldots, e_{m}\right) \rightarrow\right. \\
& \left.\qquad\left(\bigwedge_{i \leq m} \operatorname{eval}_{f}\left(e_{i}, x\right)=y_{i} \wedge \operatorname{eval}_{f}(e, x)=y \rightarrow h\left(y_{1}, \ldots, y_{m}\right)=y\right)\right] .
\end{aligned}
$$

Since we are working in the theory $T$, which does not prove the totality of eval , $_{\text {, }}$ the expression ' $\operatorname{eval}_{f}(e, x)=y$ ' is understood here as a $\Sigma_{1}$ predicate, rather than as an equality of two terms. The following two properties of this predicate can also be established within $T$ :

1. $T \vdash \forall e, i, x\left(\exists y \operatorname{eval}_{f}(e, x)=y \wedge i \leq e \rightarrow \exists y \operatorname{eval}_{f}(i, x)=y\right)$,

2. $T \vdash \forall e, x, y_{1}, y_{2}\left(\operatorname{eval}_{f}(e, x)=y_{1} \wedge \operatorname{eval}_{f}(e, x)=y_{2} \rightarrow y_{1}=y_{2}\right)$.

The first property holds because the definition of eval $f$ is constructed via the function $\overline{\mathrm{eval}}_{f}$, which presupposes a similar property. The second property follows from our proof of Proposition 4.3, because the argument for the uniqueness there requires only elementary induction.

In particular, the latter property implies that we can introduce a function symbol for eval $f$ within $\left[T, \Sigma_{1}\right.$-IR]. Thereby, inductive clauses $(*)$ can be reformulated in a more usual manner; for example, $\left[T, \Sigma_{1}\right.$-IR $]$ proves

$$
\operatorname{eval}_{f}\left(\operatorname{Sub}_{x_{0} \cdots x_{m}}\left(\ulcorner h\urcorner, e_{0}, \ldots, e_{m}\right), x\right)=h\left(\operatorname{eval}_{f}\left(e_{0}, x\right), \ldots, \operatorname{eval}_{f}\left(e_{m}, x\right)\right),
$$

for any initial function $h$. We straightforwardly obtain the following standard corollary.

Proposition 6.2. For any $f$-term $t\left(x_{1}, \ldots, x_{n}\right)$,

$$
\left[T, \Sigma_{1}-\mathrm{IR}\right] \vdash \forall x_{1}, \ldots, x_{n} \operatorname{eval}_{f}\left(\ulcorner t\urcorner,\left\langle x_{1}, \ldots, x_{n}\right\rangle\right)=t\left(x_{1}, \ldots, x_{n}\right) .
$$


Proof: induction on the buid-up of $t$, q.e.d.

To be able to more fruitfully use the inductive clauses for eval $f$ we need a reasonable amount of induction for formulas involving eval $f_{f}$. This goal is somewhat problematic, because the graph of eval $f$ is $\Sigma_{1}$, whereas the rule $\Sigma_{1}$-IR in our theory can only be applied once. Nevertheless, we have the following useful property.

Proposition 6.3. The theory $\left[T, \Sigma_{1}-\mathrm{IR}\right]$ contains the induction schema for bounded formulas in the language with a function symbol for eval $_{f}$.

Proof. The idea is to use the fact that $\left[T, \Sigma_{1}\right.$-IR $]$ is axiomatizable by monotonic $\Pi_{2}$ sentences, so that eval $f$ can be replaced by an increasing function with an elementary graph.

First, formalizing the obvious proof of Proposition 4.1 we can find a function $\operatorname{eval}_{f}^{0}$ such that eval ${ }_{f}^{0}$ has an elementary graph, the totality of eval ${ }_{f}^{0}$ is equivalent to $\mathrm{EVAL}_{f}$ over $E A$, and provably in $E A$,

$$
\operatorname{eval}_{f}(e, x)=\left(\operatorname{eval}_{f}^{0}(e, x)\right)_{1} .
$$

Now we define a function

$$
\overline{\operatorname{eval}}_{f}^{0}(x):=\max _{\langle i, j\rangle \leq x} \operatorname{eval}_{f}^{0}(i, j),
$$

whose graph is also elementary and given by the formula

$$
\begin{aligned}
& \overline{\operatorname{eval}}_{f}^{0}(x)=y: \leftrightarrow \\
& \exists\langle i, j\rangle \leq x \operatorname{eval}_{f}^{0}(i, j)=y \wedge \forall\langle i, j\rangle \leq x \exists z \leq y \operatorname{eval}_{f}^{0}(i, j)=z .
\end{aligned}
$$

We notice that the totality of $\overline{\mathrm{eval}}_{f}^{0}$, that is the formula

$$
\forall x \exists y \overline{\operatorname{eval}}_{f}^{0}(x)=y,
$$

follows from

$$
\forall x \exists y \operatorname{eval}_{f}^{0}\left((x)_{0},(x)_{1}\right)=y
$$

by an application of $\Sigma_{1}$ collection rule. The latter formula is equivalent to $\mathrm{EVAL}_{f}$, and so, by Propositions 5.3 and $5.4,(24)$ is provable in $\left[T, \Sigma_{1}\right.$-IR]. It is unproblematic to demonstrate that $\overline{\text { eval }}_{f}^{0}$ is provably functional and increasing.

We introduce a function symbol for $\overline{\text { eval }}_{f}^{0}$ into the language of $\left[T, \Sigma_{1}\right.$-IR], together with an open axiom defining the graph of $\overline{\mathrm{eval}}_{f}^{0}$. It is well-known (see [3], Proposition 1.3, page 271) that, since $\overline{\mathrm{eval}}_{f}^{0}$ is provably increasing and has an elementary graph, the resulting theory proves the induction schema for all $\Delta_{0}\left(\overrightarrow{\operatorname{eval}}_{f}^{0}\right)$ formulas, that is, for bounded formulas in the language with $\overrightarrow{\text { vall }}_{f}^{0}$. 
(Notice that such formulas precisely represent the predicates in $\mathcal{E}\left(\overline{\mathrm{eval}}_{f}^{0}\right)$, and this class coincides with $\mathbf{C}\left(\right.$ eval $\left._{f}\right)$ because the latter is elementarily closed.)

Finally we show that eval $f$ can be explicitly defined as a term in the language with a symbol for $\overline{\mathrm{eval}}_{f}^{0}$. Since $\overline{\mathrm{eval}}_{f}^{0}$ majorizes eval $f$, and eval ${ }_{f}^{0}$ has an elementary graph, eval $_{f}^{0}$ can be expressed as a composition of $\overline{\text { eval }}_{f}^{0}$ and an elementary function:

$$
\operatorname{eval}_{f}^{0}(e, x)=\mu y \leq \overline{\operatorname{eval}}_{f}^{0}(\langle e, x\rangle) . \quad y=\operatorname{eval}_{f}^{0}(e, x) .
$$

On the other hand, (22) expresses eval $f$ as a composition of an elementary function and eval $_{f}^{0}$. It follows that a bounded formula in the language with a function symbol for eval $f$ can be naturally translated into a bounded formula in the language with a symbol for $\overline{\mathrm{eval}}_{f}^{0}$, and the two formulas are provably equivalent in $\left[T, \Sigma_{1}\right.$-IR $]$. This completes the proof of Proposition 6.3, q.e.d.

Remark 6.1. Notice that we have actually proved that bounded induction for eval $_{f}$ is available in the theory $T+\overline{\mathrm{EVAL}}_{f}$, where the additional axiom asserts the totality of the function $\overline{\text { eval }}_{f}^{0}$. We do not know if, in general, $\mathrm{EVAL}_{f}$ implies $\overline{\mathrm{EVAL}}_{f}$ over $T$.

Corollary 6.4. $\left[T, \Sigma_{1}\right.$-IR] proves that for all terms $t(z)$ in one variable and all terms $u$,

$$
\forall x \quad \operatorname{eval}_{f}\left(\operatorname{Sub}_{z}(\ulcorner t\urcorner,\ulcorner u\urcorner), x\right)=\operatorname{eval}_{f}\left(\ulcorner t\urcorner,\left\langle\operatorname{eval}_{f}(\ulcorner u\urcorner, x)\right\rangle\right) .
$$

Proof: by $\Delta_{0}\left(\right.$ eval $\left._{f}\right)$ induction on the build-up of $t$, with $u$ and $x$ as free parameters, q.e.d.

\section{$7 \quad \Sigma_{1}$ induction rule}

Theorem 2. Let $T$ be an arithmetical theory containing $E A$. Then $\left[T, \Sigma_{1}-\mathrm{IR}\right]$ is equivalent to $T$ together with $\mathrm{RFN}_{\Sigma_{1}}\left(T_{0}\right)$ for all finite $\Pi_{2}$ axiomatized subtheories $T_{0}$ of $T$.

Proof. Exactly as in the proof of Theorem 1 we can show that, if for $I(x) \in \Sigma_{1}$ the theory $T$ proves

$$
I(0) \wedge \forall x(I(x) \rightarrow I(x+1)),
$$

then for a suitable finite $\Pi_{2}$ axiomatized subtheory $T_{0}$ of $T$ one has

$$
T+\operatorname{RFN}_{\Sigma_{1}}\left(T_{0}\right) \vdash \forall x I(x) .
$$

(For the axioms of $T_{0}$ one may take formula (25) together with all axioms of $E A$.)

For the opposite inclusion it is sufficient to demonstrate that

$$
\left[T, \Sigma_{1}-\mathrm{IR}\right] \vdash \mathrm{RFN}_{\Sigma_{1}}(T)
$$


for finite $\Pi_{2}$ axiomatized theories $T$. Modulo the work we have done in the previous sections the argument will be similar to the one in Sieg [18], Theorems 3.2 and 3.3 .

We introduce a function symbol $f$ for the standard witness for the single axiom of $T$ and finitely many symbols for a suitable basis in $\mathcal{E}$, so that $T$ attains a purely universal axiomatization. It is also essential that the language of $T$ is finite, and that $T$ has only finitely many nonlogical axioms in the extended language.

We know that $\left[T, \Sigma_{1}\right.$-IR] has a reasonable evaluation function eval $_{f}$ for terms in the language of $T$. Using eval $f$ first we manufacture a satisfation predicate for quantifier free formuals of $T$. The following lemma is well-known and easy.

Lemma 7.1. To every quantifier free formula $\phi(a)$ we can associate a term $\chi_{\phi}(a)$ such that

$$
T \vdash \phi(a) \leftrightarrow \chi_{\phi}(a)=0
$$

Proof. Notice that, provably in $T$,

$$
\begin{aligned}
t_{1}(a)=t_{2}(a) & \leftrightarrow\left|t_{1}(a) \dot{-} t_{2}(a)\right|=0, \\
\phi(a) \wedge \psi(a) & \leftrightarrow \chi_{\phi}(a)+\chi_{\psi}(a)=0, \\
\neg \phi(a) & \leftrightarrow 1-\chi_{\phi}(a)=0,
\end{aligned}
$$

whenever the terms $\chi_{\phi}$ and $\chi_{\psi}$ satisfy equivalence (26) for formulas $\phi$ and $\psi$. The statement of the lemma follows by induction on the build-up of $\phi$, q.e.d.

Obviously, the function

$$
\operatorname{trm}:\ulcorner\phi\urcorner \mapsto\left\ulcorner\chi_{\phi}\right\urcorner
$$

is Kalmar elementary, and Lemma 7.1 is formalizable in $E A$. We define:

$$
\operatorname{Sat}_{f}(e, a):=\left(\operatorname{eval}_{f}(\operatorname{trm}(e), a)=0\right) .
$$

This definition guarantees that $\mathrm{Sat}_{f}$ is faithfully defined on atomic formulas (by Proposition 6.2) and provably commutes with all boolean connectives. For example, provably in $\left[T, \Sigma_{1}\right.$-IR] we have: for all $\phi, \psi$,

$$
\begin{aligned}
\operatorname{Sat}_{f}(\ulcorner\phi \wedge \psi\urcorner, a) & \leftrightarrow \operatorname{eval}_{f}(\operatorname{trm}(\ulcorner\phi \wedge \psi\urcorner), a)=0 \\
& \leftrightarrow \operatorname{eval}_{f}(\operatorname{trm}(\ulcorner\phi\urcorner)+\operatorname{trm}(\ulcorner\psi\urcorner), a)=0 \\
& \leftrightarrow \operatorname{eval}_{f}(\operatorname{trm}(\ulcorner\phi\urcorner), a)+\operatorname{eval}(\operatorname{trm}(\ulcorner\psi\urcorner), a)=0 \\
& \left.\leftrightarrow \operatorname{eval}_{f}(\operatorname{trm}(\ulcorner\phi\urcorner), a)=0 \wedge \operatorname{eval}_{f}(\operatorname{trm}(\ulcorner\psi\urcorner), a)=0\right) \\
& \left.\leftrightarrow \operatorname{Sat}_{f}(\ulcorner\phi\urcorner, a) \wedge \operatorname{Sat}_{f}(\ulcorner\psi\urcorner, a)\right) .
\end{aligned}
$$

So, Tarski commutation conditions are satisfied, and in the usual manner we obtain the following lemma. 
Lemma 7.2. For every quantifier free formula $\phi\left(x_{1}, \ldots, x_{n}\right)$ in the language of $T$,

$$
\left[T, \Sigma_{1}-\mathrm{IR}\right] \vdash \forall x_{1}, \ldots, x_{n}\left(\operatorname{Sat}_{f}\left(\ulcorner\phi\urcorner,\left\langle x_{1}, \ldots, x_{n}\right\rangle\right) \leftrightarrow \phi\left(x_{1}, \ldots, x_{n}\right)\right) .
$$

We also notice the following useful property of the function trm that can be seen from our proof of Lemma 7.1: for every open formula $\phi(z)$ and every term $t$ we have:

$$
\operatorname{trm}(\ulcorner\phi(t)\urcorner)=\operatorname{Sub}_{z}(\operatorname{trm}(\ulcorner\phi\urcorner), t) .
$$

This property is formalizable in $E A$ and yields the following fact: $\left[T, \Sigma_{1}\right.$-IR] proves that for all formulas $\phi(z)$ in one variable and all terms $t$,

$$
\forall x\left(\operatorname{Sat}_{f}(\ulcorner\phi(t)\urcorner, x) \leftrightarrow \operatorname{Sat}_{f}\left(\ulcorner\phi\urcorner,\left\langle\operatorname{eval}_{f}(\ulcorner t\urcorner, x)\right\rangle\right)\right) .
$$

This essentially follows from (27) and Corollary 6.4.

Proposition 7.1. The theory $\left[T, \Sigma_{1}\right.$-IR $]$ proves the uniform reflection principle for quantifier free formulas of $T$ w.r.t. cut-free provability, that is, the following statement:

If a sequent of the form $\neg T, \Delta(a)$, where $\Delta(a)$ consists of open formulas in the language of $T$, is cut-free provable, then for all $n$, $\operatorname{Sat}_{f}(\ulcorner\bigvee \Delta\urcorner,\langle n\rangle)$.

Proof. The argument is similar to the one in the proof of Theorem 1 and, in fact, easier, although there are some subtle formal differences. Let $p$ be a cut-free derivation of a sequent of the form $(\neg T), \Delta(\bar{n})$, where $\Delta$ is quantifier free, $\bar{n}$ is any numeral, and negated axioms of $T$ possibly do not occur. Since $\neg T$ consists of purely existential formulas, w.l.o.g. we may assume that $p$ contains no free parameters. (Otherwise substitute 0 for any occurrence of a free variable in $p$.) Obviously, any subderivation $q$ of $p$ has a similar form, and its Gödel number is smaller than $p$. By induction on the height $h$ of $q$ we shall prove the following statement:

For all $h, q$, if $q$ is a subderivation of $p$ of height $h$ and the end sequent of $q$ has the form $(\neg T), \Delta^{\prime}$, then $\operatorname{Sat}_{f}\left(\left\ulcorner\bigvee \Delta^{\prime}\right\urcorner,\langle\rangle\right)$.

Since there are only finitely many subderivations of $p$, the quantifier over all $q$ in this statement is bounded, and $p$ appears as a free variable. So, the whole induction is an instance of $\Delta_{0}\left(\right.$ eval $\left._{f}\right)$ induction schema, which is available in $\left[T, \Sigma_{1}\right.$-IR] by Proposition 6.3 .

As usual, we consider several cases according to the last rule applied in the subderivation $q$. The cases of logical axioms and rules of propositional logic are easily treated using commutation properties for $\mathrm{Sat}_{f}$. The only nontrivial case is that of the existential quantifier. Suppose $\exists z \alpha(z)$ is one of the negated axioms of $T$, with $\alpha(z)$ quantifier free, and the inference has the form

$$
\frac{\neg T^{\prime},(\exists z \alpha(z)), \alpha(t), \Delta^{\prime}}{\neg T^{\prime}, \exists z \alpha(z), \Delta^{\prime}} .
$$


Then by the induction hypothesis and commutation properties for Sat $f$ we know that either $\operatorname{Sat}_{f}(\ulcorner\alpha(t)\urcorner,\langle\rangle)$ or $\operatorname{Sat}_{f}\left(\left\ulcorner\bigvee \Delta^{\prime}\right\urcorner,\langle\rangle\right)$ holds. Suppose $\operatorname{Sat}_{f}(\ulcorner\alpha(t)\urcorner,\langle\rangle)$, then by (28) we obtain $\operatorname{Sat}_{f}\left(\ulcorner\alpha(z)\urcorner,\left\langle\operatorname{eval}_{f}(t)\right\rangle\right)$, whence $\alpha\left(\operatorname{eval}_{f}(t)\right)$ by Lemma 7.2. This implies $\exists y \alpha(y)$ and a contradiction in $T$.

So, we have demonstrated $(*)$ and, considering the end sequent of the given derivation $p$, may conclude that $\operatorname{Sat}_{f}(\mathrm{~V} \Delta(\bar{n}),\langle\rangle)$ holds. By (28) this implies $\operatorname{Sat}_{f}(\mathrm{~V} \Delta,\langle n\rangle)$, q.e.d.

Now we are able to complete the proof of Theorem 2. Since $\left[E A, \Sigma_{1}\right.$-IR] contains SUPEXP and, therefore, proves the Cut-elimination Theorem for first order logic, it is sufficient to prove the $\Sigma_{1}$ reflection principle for $T$ w.r.t. cut-free provability. We reason inside $\left[T, \Sigma_{1}\right.$-IR $]$ as follows.

Suppose $\exists x \sigma(x, a)$ is cut-free provable in $T$, where $\sigma(x, a)$ is quantifier free. Since $T$ is a purely universal theory, by (formalized) Herbrand's Theorem, as in the proof of Proposition 4.2, we can find a $f$-term $t(a)$ and a cut-free $T$-derivation of the formula $\sigma(t(a), a)$. By Proposition 7.1 we may conclude that, for all $n$,

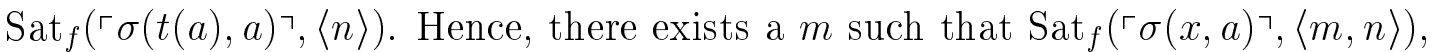
because for $m$ one can take the value of $t, \operatorname{eval}_{f}(\ulcorner t\urcorner,\langle n\rangle)$. Lemma 7.2 then yields $\exists y \sigma(y, n)$, q.e.d.

Since uniform $\Pi_{2}$ and $\Sigma_{1}$ reflection principles over $T$ are obviously equivalent, we obtain the following important corollary.

Corollary 7.1. For $\Pi_{2}$ axiomatized theories $T$ containing $E A$,

$$
\left[T, \Sigma_{1}-\mathrm{IR}\right] \equiv\left[T, \Pi_{2} \text {-IR }\right] .
$$

This corollary allows to extend to $\Sigma_{1}$-IR all the facts concerning axiomatizability that we have obtained earlier for $\Pi_{2}$ induction rule. It should be stressed, however, that these results only apply for $\Pi_{2}$ axiomatized theories, rather than for general $\Pi_{3}$ axiomatized, as in the case of $\Pi_{2}$-IR.

On the other hand, the transparent analysis of p.t.r.f.s of theories axiomatized by $\Sigma_{1}$-IR allows us to obtain nontrivial results for $\Pi_{2}$-IR. For example, we have the following result of Sieg for free (cf [19] and our discussion at the end of Section $3)$.

Corollary 7.2. The p.t.r.f.s of the theory $\left[E A, \Pi_{2}-\mathrm{IR}\right]_{k}$ are precisely those of the class $\mathcal{E}_{3+k}$ of the Grzegorczyk hierarchy.

Proof. This follows from the well-known fact (cf e.g. [16]) that classes of the Grzegorczyk hierarchy are obtained from $\mathcal{E}$ by iterated application of the operator of primitive recursion, which corresponds to $\Sigma_{1}$-IR by Proposition 4.3, q.e.d. 


\section{Relativization}

In this section we generalize the results of the previous sections to $\Sigma_{n}$-IR for an arbitrary $n$. Our main result is formulated as follows.

Theorem 3. Let $T$ be an arithmetical theory containing $I \Sigma_{n}$. Then $\left[T, \Sigma_{n+1}\right.$-IR $]$ is equivalent to $T$ together with $\mathrm{RFN}_{\Sigma_{n+1}}\left(T_{0}\right)$ for all finite $\Pi_{n+2}$ axiomatized subtheories $T_{0}$ of $T$.

Corollary 8.1. For $\Pi_{n+2}$ axiomatized theories $T$ containing $I \Sigma_{n}$,

$$
\left[T, \Sigma_{n+1}-\mathrm{IR}\right] \equiv\left[T, \Pi_{n+2^{-}} \mathrm{IR}\right] .
$$

The same result holds for $\Sigma_{n+2}$ axiomatized extensions of theories $T$ as above.

Proof. If $T_{0}$ is a finite extension of $I \Sigma_{n}$ axiomatized by a $\Pi_{n+2}$ sentence $\pi$ and a $\Sigma_{n+2}$ sentence $\sigma$, then

$$
T_{0}+\operatorname{RFN}_{\Pi_{n+2}}\left(T_{0}\right) \equiv T_{0}+\operatorname{RFN}_{\Pi_{n+2}}\left(I \Sigma_{n}+\pi\right)
$$

by formalized Deduction Theorem. And $\operatorname{RFN}_{\Pi_{n+2}}\left(I \Sigma_{n}+\pi\right)$ is provable in $\left[I \Sigma_{n}+\right.$ $\pi, \Sigma_{n+1}$-IR]. So, $\left[T, \Sigma_{n+1}\right.$-IR] proves $\operatorname{RFN}_{\Pi_{n+2}}\left(T_{0}\right)$ for any finite subtheory $T_{0}$ of $T$, exactly as $\left[T, \Pi_{n+2^{-}} \mathrm{IR}\right]$ does, q.e.d.

We see that the theorem and its corollary only apply to theories $T$ containing $I \Sigma_{n}$, rather than to arbitrary extensions of $E A$. This seems to be a fairly restrictive requirement. Notice, however, that $\left[E A, \Sigma_{n+1}-\mathrm{IR}\right]$ contains and is, in fact, equivalent to $I \Sigma_{n}$. It follows that just a single application of $\Sigma_{n+1}$-IR brings everything into the class of theories containing $I \Sigma_{n}$, where Theorem 3 applies. So, we obtain the following corollary.

Corollary 8.2. For any $\Pi_{n+2}$ axiomatized extension $T$ of $E A, k$ applications of $\Pi_{n+2}$ induction rule over $T$ are reducible to $k+1$ applications of $\Sigma_{n+1}$-IR:

$$
\left[T, \Pi_{n+2^{-}} \mathrm{IR}\right]_{k} \subseteq\left[T, \Sigma_{n+1}-\mathrm{IR}\right]_{k+1}
$$

I do not know if this result is optimal, that is, if $k+1$ applications of $\Sigma_{n+1}$-IR on the right hand side can, in general, be decreased to $k$ applications. However, we have the following result.

Corollary 8.3. Let $T$ be a $\Sigma_{n+2}$ axiomatized extension of $E A$. Then $\left[T, \Sigma_{n+1}\right.$-IR] is equivalent to $T$ together with $\mathrm{RFN}_{\Sigma_{n+1}}\left(T_{0}\right)$ for all finite subtheories $T_{0}$ of $T$. Hence, over such theories, for any $k$,

$$
\left[T, \Pi_{n+2^{-}} \mathrm{IR}\right]_{k} \equiv\left[T, \Sigma_{n+1}-\mathrm{IR}\right]_{k} .
$$


Proof. Let $T_{0}$ be a finite $\left(\Sigma_{n+2}\right.$ axiomatized $)$ subtheory of $T$. First of all, we notice that

$$
T_{0}+\mathrm{RFN}_{\Sigma_{n+1}}\left(T_{0}\right) \equiv T_{0}+\mathrm{RFN}_{\Sigma_{n+1}}(E A),
$$

by formalized Deduction Theorem. We have already noticed before that $\left[T, \Sigma_{n+1}\right.$-IR] proves $\Sigma_{n}$-IA, and by Leivant's Theorem $I \Sigma_{n}$ contains $\mathrm{RFN}_{\Sigma_{n+1}}(E A)$. (Alternatively, this fact can be seen from our proof of Theorem 3 below.) So, $\left[T, \Sigma_{n+1}\right.$-IR] proves $\operatorname{RFN}_{\Sigma_{n+1}}\left(T_{0}\right)$. The opposite inclusion is proved in the usual way.

After the first application of $\Sigma_{n+1}$-IR we obtain a theory which is a $\Sigma_{n+2}$ axiomatized extension of a $\Pi_{n+2}$ axiomatized theory containing $I \Sigma_{n}$. So, the second claim of the corollary follows by Corollary 8.1, q.e.d.

Now we turn to the proof of Theorem 3. There are several ways one can go about relativization. Our method is similar to the one employed in Sieg [18] and Ono [9], but has some additional twists. The general idea is to introduce enough Skolem functions in order to reduce Theorem 3 to 2 for a language with additional function symbols. However, in order to make this idea work one should be careful as to the choice of these Skolem functions, because the classes of functions we dealt with in the proof of Theorem 2 were not always elementarily closed.

Our first goal is to restate Theorem 2 for a language with additional function symbols. Let $\kappa(x)$ be a function. Relativized analogues of classes of functions considered in the proof of Theorem 2 are defined as follows.

$$
\begin{aligned}
\mathcal{E}^{\kappa} & :=\mathbf{E}(\kappa), \\
\mathbf{C}^{\kappa}(K) & :=\mathbf{C}\left(K \cup \mathcal{E}^{\kappa}\right) .
\end{aligned}
$$

Notice that $\mathbf{C}^{\kappa}(f)=\mathbf{C}(\bar{\kappa}, f)$, by Proposition 5.1.

Let $\Delta_{0}(\kappa)$ denote the class of bounded formulas in the language of $E A$ (with symbols for all Kalmar elementary functions) enriched by a function symbol for $\kappa$. $\Sigma_{1}^{\kappa}$ formulas are those of the form $\exists x_{1}, \ldots, x_{n} A\left(x_{1}, \ldots, x_{n}, a\right)$, where $A \in \Delta_{0}(\kappa)$. Classes $\Sigma_{n}^{\kappa}$ and $\Pi_{n}^{\kappa}$ are defined in a similar manner.

Relativized version of Kalmar elementary arithmetic, $E A^{\kappa}$, is a theory formulated in the language with a function symbol for $\kappa$. In addition to the usual axioms of $E A$ it has a schema of induction for $\Delta_{0}(\kappa)$ formulas. This formulation of $E A^{\kappa}$ is not purely universal because of the presence of bounded quantifiers. We show how to reformulate it in a purely universal way.

First of all, we show that one can naturally $\Delta_{0}(\kappa)$ define the graph of $\bar{\kappa}$ and prove in $E A^{\kappa}$ that this relation defines a total function. For example, one can first define an auxiliary function $t(x)$ by

$$
t(x):=\mu z \leq x . \forall i \leq x \kappa(i) \leq \kappa(z) .
$$

The graph of $t$ is clearly $\Delta_{0}(\kappa)$, and since $t(x) \leq x$ holds provably in $E A^{\kappa}$, the totality of $t$ is easily proved by $\Delta_{0}(\kappa)$ induction. So, we introduce a function 
symbol for $t$ and then define $m(x):=\kappa(t(x))$. It is easy to see, provably in $E A^{\kappa}$, that

$$
m(x)=\max _{i \leq x} \kappa(x) .
$$

Now we define the graph of $\bar{\kappa}$ as follows:

$$
\bar{\kappa}(x)=y: \leftrightarrow y \in \operatorname{Seq} \wedge \operatorname{lh}(y)=x+1 \wedge \forall i \leq y(y)_{i}=\kappa(i),
$$

where $\ln (y)$ denotes the length of a sequence $y$. To show that

$$
\forall x \exists y \bar{\kappa}(x)=y
$$

we notice that

$$
\bar{\kappa}(x)=\mu y \cdot y \in \operatorname{Seq} \wedge \operatorname{lh}(y)=x+1 \wedge \forall i \leq x \kappa(i) \leq(y)_{i}
$$

So, given a $x$ we can find a sequence $y=\langle m(x), \ldots, m(x)\rangle$ that majorizes $\kappa$ on the interval $[0, x]$. Then we apply $\Delta_{0}(\kappa)$ least element principle to find the minimal such $y$. This proves (29).

The following two useful properties of the function $\bar{\kappa}$ are obviously provable in $E A^{\kappa}$.

1. $\forall x(\bar{\kappa}(x) \in \operatorname{Seq} \wedge \operatorname{lh}(\bar{\kappa}(x))=x+1)$,

2. $\forall x, y\left(x \leq y \rightarrow \bar{\kappa}(x) \subseteq_{e} \bar{\kappa}(y)\right)$.

Here $x \subseteq_{e} y$ means that $x$ is an initial segment of a sequence $y$. In particular, the second property shows that $\bar{\kappa}$ is a provably increasing function. By [3] (Proposition 1.3, page 271) we know that for such functions $\Delta_{0}(\bar{\kappa})$ induction is reducible (over $E A$ ) to induction for predicates elementary in the graph of $\bar{\kappa}$, i.e., for formulas built up from $\bar{\kappa}(x)=y$ and elementary ones using boolean connectives and quantifiers bounded by elementary functions. Since the graph of $\bar{\kappa}$ is $\Delta_{0}(\kappa)$, we see that $\Delta_{0}(\bar{\kappa})$ induction schema is available in $E A^{\kappa}$.

On the other hand, let $E A^{\bar{\kappa}}$ be a theory formulated in the language of $E A$ enriched by a function symbol for $\bar{\kappa}$. Axioms of $E A^{\bar{\kappa}}$ are those of $E A$ plus induction schema for open formulas plus formulas 1 and 2 above. We have just seen that it is contained, or rather interpreted, in $E A^{\kappa}$. The opposite containment is also true.

Proposition 8.1. $E A^{\kappa}$ is equivalent to $E A^{\bar{\kappa}}$.

Proof. First of all, formalizing the proof of Proposition 4.2 we can show that $\mathbf{C}(\bar{\kappa})$ is provably closed under bounded summation.

Lemma 8.1. For every term $g(x, a)$ in the language of $E A^{\bar{\kappa}}$ we can (effectively) find a term $G(x, a)$ such that $E A^{\bar{\kappa}}$ proves:

$$
\begin{aligned}
G(0, a) & =g(0, a), \\
G(x+1, a) & =G(x, a)+g(x+1, a) .
\end{aligned}
$$


Notice that any two terms satisfying the above equations are provably equal in $E A^{\bar{\kappa}}$. We shall denote $G(x, a)$ by $\sum_{i \leq x} g(i, a)$. A similar lemma holds for bounded multiplication.

Lemma 8.2. For every $\Delta_{0}(\bar{\kappa})$ formula $\phi(a)$ there is a term $\chi_{\phi}(a)$ such that

$$
E A^{\bar{\kappa}} \vdash \forall x\left(\phi(x) \leftrightarrow \chi_{\phi}(x)=0\right) .
$$

Proof. Induction on the build up of $\phi$. Boolean connectives are treated as in Lemma 7.1. Bounded quantifiers are translated using Lemma 8.1 as follows:

$$
\forall x \leq y \phi(x, a) \leftrightarrow \sum_{x \leq y} \chi_{\phi}(x, a)=0,
$$

whenever $\chi_{\phi}$ satisfies the induction hypothesis. We only need to demonstrate equivalence (30) in $E A^{\bar{\kappa}}$ using open induction.

For the implication $(\leftarrow)$ we prove

$$
\sum_{x \leq y} \chi_{\phi}(x, a)=0 \wedge u \leq y \rightarrow \chi_{\phi}(u, a)=0
$$

by an obvious quantifier free induction on $y$. For the opposite implication $(\rightarrow)$ we reason as follows. Assume $\forall x \leq y \phi(x, a)$. Then prove by quantifier free induction on $u$, and with $y$ a parameter, that

$$
\forall u \leq y \sum_{x \leq u} \chi_{\phi}(x, a)=0 .
$$

Conclude $\sum_{x \leq y} \chi_{\phi}(x, a)=0$. Notice that the induction here, being applied as a schema, does not involve the side formula $\forall x \leq y \phi(x, a)$ (which is not quantifier free), q.e.d.

¿From Lemma 8.2 it follows that, using open induction only, we can prove all instances of $\Delta_{0}(\bar{\kappa})$ induction in $E A^{\bar{\kappa}}$. Now we notice that the function $\kappa$ can be defined by a term in $E A^{\bar{k}}$ :

$$
\kappa(x):=(\bar{\kappa}(x))_{x} .
$$

This means that $\Delta_{0}(\kappa)$ induction is reducible to $\Delta_{0}(\bar{\kappa})$ induction, and we may conclude that $E A^{\kappa}$ is equivalent to $E A^{\bar{\kappa}}$, since the two interpretations we constructed are mutually inverse, q.e.d.

Proposition 8.2. $E A^{\bar{\kappa}}$ has a purely universal axiomatization (in the language with symbols for $\bar{\kappa}$ and for all elementary functions).

Proof. In the standard axiomatization of $E A^{\bar{\kappa}}$ the instances of quantifier free induction

$$
A(0) \wedge \forall y \leq x(A(x) \rightarrow A(x+1)) \rightarrow \forall y \leq x A(y)
$$


are bounded, but not literally quantifier free. We show that in an axiomatization of $E A^{\bar{\kappa}}$ these formulas can be replaced by quantifier free ones. To this end we have to improve a little upon Lemma 8.2. We show that in the proof of Lemma 8.2 only a number of purely universal theorems of $E A^{\bar{\kappa}}$ could be used.

Indeed, the treatment of boolean connectives in Lemma 7.1 only requires a finite number of equivalences, like

$$
|x-y|=0 \leftrightarrow x=y,
$$

or

$$
x+y=0 \leftrightarrow(x=0 \wedge y=0) .
$$

To handle the bounded quantifiers we can simply take open formulas (31) as axioms. However, the proof of the implication

$$
\forall x \leq y \chi_{\phi}(x, a) \rightarrow \sum_{x \leq y} \chi_{\phi}(x, a)=0,
$$

poses a problem.

Let $m(y, a)$ be a function defined by

$$
m(y, a):=\mu x \leq y \cdot \chi_{\phi}(x, a) \neq 0 .
$$

It is well-known that $m(y, a)$ belongs to $\mathcal{E}^{\bar{\kappa}}$ (cf Rose [14]), and hence, is definable via bounded summation and multipliction. Moreover, in $E A^{\bar{\kappa}}$ one can prove natural properties of $\mu$ operator by quantifier free induction, in particular,

$$
\sum_{x \leq y} \chi_{\phi}(x, a) \neq 0 \rightarrow \chi_{\phi}(m(y, a), a) \neq 0
$$

is provable in $E A^{\bar{\kappa}}$. This formula clearly implies (32), and so, we can take it as another open axiom. Thus, we see that Lemma 8.2 follows from a number of purely universal theorems of $E A^{\bar{\kappa}}$. Taking these theorems together with open translations of all instances of quantifier free induction yields an open axiomatization of $E A^{\bar{\kappa}}$, q.e.d.

Let us also note that Lemma 8.2 and Proposition 8.2 actually show that, in order to quantifier free represent any individual $\Delta_{0}(\bar{\kappa})$ formula one only needs a finite fragment of $E A^{\bar{\kappa}}$, i.e., finitely many (Kalmar elementary) function symbols and finitely many open $E A^{\bar{\kappa}}$ axioms for them. We shall use this fact later, for we do not want to be involved in a rather technical proof of finite axiomatizability of the whole of $E A^{\bar{\kappa}}$.

Now we can formulate a relativized version of (a particular case of) Theorem 2.

Theorem 4. Let $T$ be a finite $\Pi_{2}^{\bar{\kappa}}$ axiomatized theory. Then

$$
\left[E A^{\bar{\kappa}}+T, \Sigma_{1}^{\bar{\kappa}} \mathrm{IR}\right] \vdash \mathrm{RFN}_{\Sigma_{1}^{\bar{\kappa}}}(T) .
$$


Proof. We check that everything in the proof of Theorem 2 relativizes. (Notice that the relativized theorem is formulated in such a way that finite axiomatizability of $E A^{\bar{\kappa}}$ is not used.) We take a purely universal formulation of $E A^{\bar{\kappa}}$ and introduce a new function symbol $f$ for the standard witness of the (single) $\Pi_{2}^{\bar{\kappa}}$ axiom of $T$. At the cost of introducing into the language of $T$ finitely many function symbols for elementary (in $\bar{\kappa}$ ) functions, and adding finitely many purely universal axioms of $E A^{\bar{\kappa}}$ we may assume that the graph of $f$ is open and $T$ has a finite purely universal axiomatization in the language with $f$.

Main steps in the proof of Theorem 2 were as follows. (a) Defining the evaluation function for $f$-terms using only one primitive recursion over $\mathbf{C}(f)$; (b) Proving the totality and natural commutation properties for eval $f$ inside $\left[T, \Sigma_{1}\right.$-IR]; (c) Showing that $\Delta_{0}\left(\right.$ eval $\left._{f}\right)$ induction schema is available in $\left[E A^{\bar{\kappa}}+T, \Sigma_{1}\right.$-IR $]$; (d) Proving uniform reflection principle for open formulas of $T$ (in the language with $f)$ by $\Delta_{0}\left(\right.$ eval $\left._{f}\right)$ induction.

Step (a) relativizes simply because $\mathbf{C}^{\bar{\kappa}}(f)=\mathbf{C}(\bar{\kappa}, f)$. So, relativizing the first part of Proposition 4.3 we obtain a $\Sigma_{1}^{\bar{\kappa}}$ definition of the evaluation function $\operatorname{eval}_{f}^{\bar{\kappa}}(e, x)$ for terms in the language of $T$, and show its totality and natural properties in $\left[E A^{\bar{\kappa}}+T, \Sigma_{1}^{\bar{\kappa}}\right.$-IR]. This only depends on the assumption that $f$ has a $\Delta_{0}(\bar{\kappa})$ graph and that $E A^{\bar{\kappa}}$ contains enough $\Delta_{0}(\bar{\kappa})$ induction. This accounts for (b).

The proof of (c) depends on the results of Section 5, most notably on Lemma 5.4. These results relativize as they are, using the fact that the function $\bar{\kappa}$ is provably increasing. Then the proof of relativized Proposition 6.3 is also unproblematic. This gives us a natural satifaction predicate in $\left[E A^{\bar{\kappa}}+T, \Sigma_{1}^{\bar{\kappa}}\right.$-IR $]$ for quantifier free formulas of $T$, $\operatorname{Sat}_{f}^{\bar{k}}(e, x)$. The proof of $(\mathrm{d})$ is no different from Proposition 7.1. Here we essentially rely on the fact that $T$ is a finite and purely universal theory, q.e.d.

Remark 8.1. Obviously, the analog of Theorem 4 also holds for extensions of the language of arithmetic by more then one additional function symbol $\bar{\kappa}$.

Proof of Theorem 3. For the sake of clarity of presentation we only give a proof of this theorem for $n=1$. For larger $n$ the proof is very similar.

Let $T$ be a finite extension of $I \Sigma_{1}$ with the only $\Pi_{3}$ axiom

$$
\tau:=\forall u \exists v \forall w \tau_{0}(u, v, w),
$$

with $\tau_{0}$ bounded. At the cost of introducing finitely many (Kalmar elementary) function symbols and purely universal axioms for them we may assume that $\tau_{0}$ is open. Let

$$
\phi(x):=\exists u \forall v \theta(u, v, x)
$$

be an arbitrary $\Sigma_{2}$ formula. Again, we may assume $\theta$ open, possibly slightly increasing the language of $T$. We are going to show that

$$
\left[T, \Sigma_{2} \text { IR }\right] \vdash \forall x\left(\operatorname{Prov}_{T}(\ulcorner\phi(\dot{x})\urcorner) \rightarrow \phi(x)\right) .
$$


To this end first we introduce Skolem functions in order to eat up the innermost universal quantifiers in $\tau$ and $\phi$, i.e., new function symbols $\kappa(u, x)$ and $\nu(u, v)$ together with the axioms:

$$
\begin{gathered}
\theta(u, \kappa(u, x), x) \rightarrow \theta(u, v, x), \\
\tau_{0}(u, v, \nu(u, v)) \rightarrow \tau_{0}(u, v, w) .
\end{gathered}
$$

Clearly, axioms (33) and (34) logically imply

$$
\begin{aligned}
\tau & \leftrightarrow \forall u \exists v \tau_{0}(u, v, \nu(u, v)) \text { and } \\
\phi(x) & \leftrightarrow \exists u \theta(u, \kappa(u, x), x) .
\end{aligned}
$$

Let $T^{+}$be a theory obtained by adding to $T$ axioms (33) and (34). Obviously, $T^{+}$has a finite $\Pi_{2}^{\kappa, \nu}$ axiomatization, and $\phi(x)$ is equivalent in $T^{+}$to a $\Sigma_{1}^{\kappa}$ formula.

Lemma 8.3. There is an interpretation $(\cdot)^{-}$of $E A^{\bar{\kappa}, \bar{\nu}}+T^{+}$in $T$ such that:

(a) $(\cdot)^{-}$is identical on formulas in the language of $T$;

(b) If $A \in \Sigma_{1}^{\bar{\kappa}, \bar{\nu}}$ then $(A)^{-}$is equivalent to a $\Sigma_{2}$ formula in $T$.

Proof. The graphs of $\nu$ and $\kappa$ can be defined as follows:

$$
\begin{array}{ll}
(\kappa(u, x)=y)^{-} & : \leftrightarrow \quad(\neg \theta(u, y, x) \wedge \forall w<y \theta(u, w, x)) \vee(\forall z \theta(u, z, x) \wedge y=0) \\
(\nu(u, v)=y)^{-} & : \leftrightarrow \quad\left(\neg \tau_{0}(u, v, y) \wedge \forall w<y \tau_{0}(u, v, w)\right) \vee(\forall z \theta(u, v, z) \wedge y=0) .
\end{array}
$$

Notice that graphs of $\kappa$ and $\nu$ are $\Pi_{1}$ under this interpretation, and that $E A$ proves that $\kappa$ and $\nu$ are total functions (although, in general, they are not - and cannot be - recursive).

It is easy to check that interpretations of the axioms (33) and (34) are provable in $E A$. The strength of $I \Sigma_{1}$ is needed to prove the interpreted axioms of $E A^{\bar{\kappa}, \bar{\nu}}$. We have already noticed that $E A^{\bar{\kappa}, \bar{\nu}}$ can be axiomatized by induction schema for bounded formulas in the graphs of $\bar{\kappa}$ and $\bar{\nu}$. Such formulas are naturally translated into $\Delta_{0}\left(\Pi_{1}\right)$ formulas, and $\Delta_{0}\left(\Pi_{1}\right)$ induction schema is available in $I \Sigma_{1}$. This proves that $(\cdot)^{-}$is the required interpretation. Property (a) is obvious, and for (b) it is sufficient to demonstrate that $\Delta_{0}(\bar{\kappa}, \bar{\nu})$ formulas are $\Delta_{2}$ in $T$ under the interpretation in question. By Lemma 8.2 every $\Delta_{0}(\bar{\kappa}, \bar{\nu})$ formula is equivalent to an open formula in $E A^{\bar{\kappa}, \bar{\nu}}$. Such formulas are obviously equivalent in $E A^{\bar{\kappa}, \bar{\nu}}$ to $\Delta_{1}$ formulas in the graphs of $\bar{\kappa}$ and $\bar{\nu}$. Since the graphs of $\bar{\kappa}$ and $\bar{\nu}$ are $\Pi_{1}$, interpretations of these formulas are $\Delta_{2}$ in $T$, q.e.d.

An obvious corollary of this lemma is the fact that $T^{+}$is a conservative extension of $T$, and this fact is provable in $E A$ (because $T^{+}$is a finite theory). Now we are in a position to invoke Theorem 4. Since $T^{+}$is finite and $\Pi_{2}^{\kappa, \nu}$ axiomatized, we have

$$
\left[E A^{\bar{\kappa}, \bar{\nu}}+T^{+}, \Sigma_{1}^{\bar{\kappa}, \bar{\nu}}-\mathrm{IR}\right] \vdash \forall x\left(\operatorname{Prov}_{T^{+}}(\ulcorner\exists u \theta(u, \kappa(u, \dot{x}), \dot{x})\urcorner) \rightarrow \exists u \theta(u, \kappa(u, x), x)\right) .
$$


Since

$$
T^{+} \vdash \phi(x) \leftrightarrow \exists u \theta(u, \kappa(u, x), x),
$$

by the provable conservativity of $T^{+}$over $T$ it follows that

$$
E A \vdash \operatorname{Prov}_{T^{+}}(\ulcorner\exists u \theta(u, \kappa(u, \dot{x}), \dot{x})\urcorner) \leftrightarrow \operatorname{Prov}_{T}(\ulcorner\phi(\dot{x})\urcorner),
$$

and therefore

$$
\left[E A^{\bar{\kappa}, \bar{\nu}}+T^{+}, \Sigma_{1}^{\bar{\kappa}, \bar{\nu}}-\mathrm{IR}\right] \vdash \forall x\left(\operatorname{Prov}_{T}(\ulcorner\phi(\dot{x})\urcorner) \rightarrow \phi(x)\right) .
$$

So, we can find $\Sigma_{1}^{\bar{\kappa}, \bar{\nu}}$ formulas $I_{1}(x), \ldots, I_{k}(x)$ such that, for each $i$,

$$
E A^{\bar{\kappa}, \bar{\nu}}+T^{+} \vdash I_{i}(0) \wedge \forall x\left(I_{i}(x) \rightarrow I_{i}(x+1)\right),
$$

and

$$
E A^{\bar{\kappa}, \bar{\nu}}+T^{+}+\left\{\forall x I_{i}(x) \mid i=1, \ldots, k\right\} \vdash \forall x\left(\operatorname{Prov}_{T}(\ulcorner\phi(\dot{x})\urcorner) \rightarrow \phi(x)\right) .
$$

Since $(\cdot)^{-}$, being an interpretation, distributes over boolean connectives and quantifiers, from $(35)$ we obtain $\Sigma_{2}$ formulas $I_{1}^{-}(x), \ldots, I_{k}^{-}(x)$ such that

$$
T \vdash I_{i}^{-}(0) \wedge \forall x\left(I_{i}^{-}(x) \rightarrow I_{i}^{-}(x+1)\right),
$$

for all $i$. And (36) implies that

$$
T+\left\{\forall x I_{i}^{-}(x) \mid i=1, \ldots, k\right\} \vdash \forall x\left(\operatorname{Prov}_{T}(\ulcorner\phi(\dot{x})\urcorner) \rightarrow \phi(x)\right),
$$

so we obtain

$$
\left[T, \Sigma_{2} \text { IR }\right] \vdash \forall x\left(\operatorname{Prov}_{T}(\ulcorner\phi(\dot{x})\urcorner) \rightarrow \phi(x)\right) .
$$

This finishes the proof of the main part of Theorem 3. The other part is no different from that of Theorem 2, q.e.d.

\section{Conclusion}

In this paper we introduced natural notions of reducibility and congruence of rules in formal arithmetic. We classified various forms of induction rules of restricted arithmetical complexity (over $E A$ ) modulo congruence relation. It turned out that these forms, most commonly, fall into one of the three main (distinct) categories: (a) rules congruent to induction axiom schemata; (b) rules congruent to $\Sigma_{n}$ induction rule $\Sigma_{n}$-IR; (c) rules congruent to $\Pi_{n}$ induction rule $\Pi_{n}$-IR.

We gave characterizations of $\Sigma_{n}$-IR and $\Pi_{n}$-IR in terms of iterated reflection principles. These characterizations provide natural axiomatizations for closures of arbitrary theories containing $E A$ under these rules. It turns out that the number of iterations of reflection principles precisely corresponds to the depth of 
nestings of applications of induction rules. This shows, in particular, that the two ways of axiomatizing theories are tightly related.

Besides, these characterizations yield several important corollaries concerning finite (non)axiomatizability of theories axiomatized by induction rules, optimal complexity of their axiomatizations, and give wide sufficient conditions for the equivalence of (closures of theories by) $\Pi_{n+1}$ and $\Sigma_{n}$ induction rules.

Proof-theoretic analysis of provably recursive functions of theories axiomatized by rules allows us to sharpen, and give easy new proofs of, several old results. For example, we prove Peter's theorem on reduction of nested recursion to primitive recursion and Finite Basis Theorem for Kalmar elementary functions. We also reproduce some results of Parsons [12] and Sieg [18, 19], e.g., we show that Parsons' result on $\Pi_{2}$ conservativity of $I \Sigma_{1}$ over $\Sigma_{1}$-IR is interderivable with (a particular case of) so-called Fine Structure Theorem on uniform reflection principles of U.Schmerl [15].

I hope the results of this paper will convince the reader of the fact that rules in arithmetic are an interesting independent object of study; and that a detailed analysis how particular rules work not only often reveals peculiar effects, but may have useful applications in other topics of proof theory.

\section{References}

[1] M. Baaz and P. Pudlák. Kreisel's conjecture for $L \exists_{1}$. In P. Clote and J. Krajíček, editors, Arithmetic, Proof Theory, and Computational Complexity, pages 29-59. Oxford University Press, Oxford, 1993. With a postscript by G. Kreisel.

[2] L.D. Beklemishev. On a restricted induction rule and iterated reflection principles over Kalmar elementary arithmetic. In O.B. Lupanov, editor, Theoretical and applied aspects of mathematical investigations, pages 36-39. Moscow State University, Moscow, 1994. In Russian.

[3] P. Hájek and P. Pudlák. Metamathematics of First Order Arithmetic. Springer-Verlag, Berlin, Heidelberg, New-York, 1993.

[4] U. Kohlenbach. A note on $\Pi_{2}^{0}$-induction rule. Archive for Mathematical Logic, 1995.

[5] J. Krajíček. Bounded arithmetic, Propositional logic, and Complexity theory. Oxford University Press, Oxford. To appear.

[6] G. Kreisel and A. Lévy. Reflection principles and their use for establishing the complexity of axiomatic systems. Zeitschrift f. math. Logik und Grundlagen d. Math., 14:97-142, 1968. 
[7] D. Leivant. The optimality of induction as an axiomatization of arithmetic. Journal of Symbolic Logic, 48:182-184, 1983.

[8] A.A. Muchnik. On two approaches to the classification of recursive functions. In A.A. Muchnik and V.A. Kozmidiadi, editors, Problems in Mathematical Logic: Complexity of Algorithms and Classes of Computable Functions, pages 123-138. MIR, Moscow, 1970. In Russian.

[9] H. Ono. Reflection principles in fragments of Peano Arithmetic. Zeitschrift f. math. Logik und Grundlagen d. Math., 33(4):317-333, 1987.

[10] C. Parsons. Hierarchies of primitive recursive functions. Zeitschrift f. math. Logik und Grundlagen d. Math., 14(4):357-376, 1968.

[11] C. Parsons. On a number-theoretic choice schema and its relation to induction. In Kino, Myhill, and Vessley, editors, Intuitionism and Proof Theory, pages 459-473. North Holland, Amsterdam, 1970.

[12] C. Parsons. On n-quantifier induction. Journal of Symbolic Logic, 37(3):466482, 1972.

[13] R. Péter. Recursive Functions. Academic Press, 1967.

[14] H.E. Rose. Subrecursion: Functions and Hierarchies. Clarendon Press, Oxford, 1984.

[15] U.R. Schmerl. A fine structure generated by reflection formulas over Primitive Recursive Arithmetic. In M. Boffa, D. van Dalen, and K. McAloon, editors, Logic Colloquium'78, pages 335-350. North Holland, Amsterdam, 1979 .

[16] H. Schwichtenberg. Rekursionszahlen und die Grzegorczyk-Hierarchie. Archive for Mathematical Logic, 12:85-97, 1969.

[17] H. Schwichtenberg. Some applications of cut-elimination. In J. Barwise, editor, Handbook of Mathematical Logic, pages 867-896. North Holland, Amsterdam, 1977.

[18] W. Sieg. Fragments of arithmetic. Annals of Pure and Applied Logic, 28:33$71,1985$.

[19] W. Sieg. Herbrand analyses. Archive for Mathematical Logic, 30:409-441, 1991.

[20] W. Sieg and S. Wainer. Program transformation and proof transformation. Typeset manuscript, 1995. 
[21] C. Smoryński. The incompleteness theorems. In J. Barwise, editor, Handbook of Mathematical Logic, pages 821-865. North Holland, Amsterdam, 1977.

[22] A. Wilkie and J. Paris. On the scheme of induction for bounded arithmetic formulas. Annals of Pure and Applied Logic, 35:261-302, 1987. 